\title{
Global Schauder estimates for a class of degenerate Kolmogorov equations
}

by

\author{
EnRICO PRIOLA (Torino)
}

\begin{abstract}
We consider a class of possibly degenerate second order elliptic operators $\mathcal{A}$ on $\mathbb{R}^{n}$. This class includes hypoelliptic Ornstein-Uhlenbeck type operators having an additional first order term with unbounded coefficients. We establish global Schauder estimates in Hölder spaces both for elliptic equations and for parabolic Cauchy problems involving $\mathcal{A}$. The Hölder spaces in question are defined with respect to a possibly nonEuclidean metric related to the operator $\mathcal{A}$. Schauder estimates are deduced by sharp $L^{\infty}-C^{\theta}$ estimates on the spatial derivatives of the associated diffusion semigroup which are of independent interest. In the proof we also use probabilistic techniques.
\end{abstract}

1. Introduction. Let us consider the following possibly degenerate second order elliptic operator $\mathcal{A}$ on $\mathbb{R}^{n}$ :

$$
\begin{aligned}
\mathcal{A} u(x) & =\frac{1}{2} \operatorname{Tr}\left(Q D^{2} u(x)\right)+\langle A x, D u(x)\rangle+\langle F(x), D u(x)\rangle \\
& =\mathcal{A}_{0} u(x)+\langle F(x), D u(x)\rangle, \quad x \in \mathbb{R}^{n} .
\end{aligned}
$$

Here $Q$ and $A$ are $n \times n$ real matrices, $Q$ is symmetric and non-negative definite, $\operatorname{Tr}(\cdot)$ denotes the trace and $\langle\cdot, \cdot\rangle$ the inner product in $\mathbb{R}^{n}$. Furthermore, $F: \mathbb{R}^{n} \rightarrow \mathbb{R}^{n}$ is a regular possibly unbounded vector field. The operator $\mathcal{A}$ in the special case $F=0$ reduces to the extensively studied possibly degenerate Ornstein-Uhlenbeck operator $\mathcal{A}_{0}$. Degenerate Kolmogorov operators like $\mathcal{A}$ arise in kinetic theory (see [8] and the references therein) and in mathematical finance (see the survey paper [9]). Following [8], consider the linear Fokker-Planck equation

$$
\partial_{t} h=\left\langle v, D_{x} h\right\rangle+\operatorname{div}_{v}\left(D_{v} h+v h\right),
$$

where $h(t, x, v)$ is the density of particles at position $x \in \mathbb{R}^{d}$ at time $t \geq 0$ with velocity $v \in \mathbb{R}^{d}$. The degenerate operator $\mathcal{B}$ given by $\mathcal{B} h=\left\langle v, D_{x} h\right\rangle+$ $\operatorname{div}_{v}\left(D_{v} h+v h\right)-d h$, is a particular case of $\mathcal{A}$ occurring when $\mathbb{R}^{n}=\mathbb{R}^{2 d}$. We also mention that operators like $\mathcal{A}$ arise naturally in connection with the

2000 Mathematics Subject Classification: 35K65, 35B65, 35J70, 47D07, 60J35.

Key words and phrases: Schauder estimates, degenerate elliptic and parabolic equations, Kolmogorov equations, hypoelliptic operators, diffusion semigroups. 
so-called Smoluchowski-Kramers approximation for stochastic differential equations (see [13]).

The aim of the present paper is to prove global Schauder estimates for elliptic equations and parabolic Cauchy problems involving the operator $\mathcal{A}$. We obtain optimal regularity results in Hölder spaces for both

$$
\lambda u(x)-\mathcal{A} u(x)=f(x), \quad x \in \mathbb{R}^{n},
$$

and

$$
\left\{\begin{aligned}
\partial_{t} v(t, x) & =\mathcal{A} v(t, x)+H(t, x), \quad t \in(0, T], x \in \mathbb{R}^{n}, \\
v(0, x) & =g(x), \quad x \in \mathbb{R}^{n},
\end{aligned}\right.
$$

where $\lambda>0$ and the functions $f, g$ and $H$ are given. These results are deduced by sharp $L^{\infty}$-estimates on the spatial derivatives of the solution of (1.3) when $H=0$, involving Hölder norms of the initial datum $g$.

Global Schauder estimates have been used recently in connection with stochastic differential equations (see $[1,6,11]$ ). In [6] Schauder estimates for degenerate elliptic operators $L$ in non-smooth domains are a key ingredient to investigate well-posedness of the martingale problem associated to $L$. In [11] parabolic Schauder estimates are used to prove the existence of a differentiable stochastic flow in the case of stochastic differential equations with Hölder continuous drift term.

Let us collect our assumptions on the operator $\mathcal{A}$ (cf. [29]).

Hypothesis 1.1. (i) The symmetric matrix $Q=\left(q_{i j}\right)_{i, j=1}^{n}$ is given by $Q=\left(\begin{array}{cc}Q_{0} & 0 \\ 0 & 0\end{array}\right)$, where

$Q_{0}$ is a positive definite $\tilde{p} \times \tilde{p}$ matrix, $1 \leq \tilde{p} \leq n$;

$\nu_{1}$ and $\nu_{2}$ stand for the smallest and the largest eigenvalue of $Q_{0}$ respectively $\left(0<\nu_{1} \leq \nu_{2}\right)$;

(ii) the vector field $F: \mathbb{R}^{n} \rightarrow \mathbb{R}^{n}$ has the form

$$
F(x)=\left(F_{1}(x), \ldots, F_{\tilde{p}}(x), 0, \ldots, 0\right), \quad x \in \mathbb{R}^{n},
$$

i.e., $F(x) \in \operatorname{Im}(Q)$ for any $x \in \mathbb{R}^{n}$;

(iii) the non-zero coefficients of $F$, i.e., $F_{i}: \mathbb{R}^{n} \rightarrow \mathbb{R}, i=1, \ldots, \tilde{p}$, are Lipschitz continuous having continuous and bounded partial derivatives up to the third order on $\mathbb{R}^{n}$;

(iv) there exists a non-negative integer $k$ such that the vectors

$$
\left\{e_{1}, \ldots, e_{\tilde{p}}, A e_{1}, \ldots, A e_{\tilde{p}}, \ldots, A^{k} e_{1}, \ldots, A^{k} e_{\tilde{p}}\right\} \text { generate } \mathbb{R}^{n} ;
$$

here $e_{1}, \ldots, e_{\tilde{p}}$ are the first $\tilde{p}$ elements of the canonical basis in $\mathbb{R}^{n}$; we denote by $k$ the smallest non-negative integer such that (1.5) holds (one has $0 \leq k \leq n-1)$. 
Condition (1.5) can also be written as $\operatorname{Rank}\left[Q^{1 / 2}, A Q^{1 / 2}, \ldots, A^{k} Q^{1 / 2}\right]$ $=n$. By the Hörmander condition on commutators, (1.5) is equivalent to the hypoellipticity of the operator $\mathcal{A}_{0}-\partial_{t}$ in $n+1$ variables $\left(t, x_{1}, \ldots, x_{n}\right)$; see [18]. Our operator $\mathcal{A}$ has the form $\mathcal{A} u(x)=\frac{1}{2} \sum_{i, j=1}^{\tilde{p}} q_{i j} \partial_{x_{i} x_{j}}^{2} u(x)+\sum_{i=1}^{\tilde{p}} F_{i}(x) \partial_{x_{i}} u(x)+\sum_{i, j=1}^{n} a_{i j} x_{j} \partial_{x_{i}} u(x), \quad x \in \mathbb{R}^{n}$, where the $a_{i j}$ are the components of the matrix $A$ and $\partial_{x_{i}}$ and $\partial_{x_{i} x_{j}}^{2}$ are partial derivatives. Clearly, the operator $\mathcal{A}$ is non-degenerate only when $\tilde{p}=n$ (this implies $k=0$ ).

Let us describe our results for (1.2) and (1.3). In the elliptic equation (1.2) we assume that $f \in \mathcal{C}_{d}^{\theta}\left(\mathbb{R}^{n}\right), \theta \in(0,1)$, i.e., $f$ is a real bounded function on $\mathbb{R}^{n}$, which is Hölder continuous with respect to a suitable possibly nonEuclidean metric $d$ related to $\mathcal{A}$ (see Section 2). We show that (1.2) has a unique bounded distributional solution $u \in \mathcal{C}_{d}^{2+\theta}\left(\mathbb{R}^{n}\right)$ and, furthermore, that there exists a positive constant $C$, independent of $f$ and $u$, such that $\|u\|_{\mathcal{C}_{d}^{2+\theta}\left(\mathbb{R}^{n}\right)} \leq C\|f\|_{\mathcal{C}_{d}^{\theta}\left(\mathbb{R}^{n}\right)}$. Note that this implies

$$
\|u\|_{0}+\sum_{i, j=1}^{\tilde{p}}\left\|\partial_{x_{i} x_{j}}^{2} u\right\|_{\mathcal{C}_{d}^{\theta}\left(\mathbb{R}^{n}\right)} \leq C\|f\|_{\mathcal{C}_{d}^{\theta}\left(\mathbb{R}^{n}\right)},
$$

where $\|u\|_{0}$ denotes the sup-norm of $u$ and $\partial_{x_{i} x_{j}}^{2} u$ are the classical partial derivatives of $u$ for $i, j=1, \ldots, \tilde{p}$ (see Theorem 4.2). Concerning the Cauchy problem (1.3) we prove analogous parabolic Schauder estimates, assuming that $g \in \mathcal{C}_{d}^{2+\theta}\left(\mathbb{R}^{n}\right)$ and $H(t, \cdot) \in \mathcal{C}_{d}^{\theta}\left(\mathbb{R}^{n}\right)$ uniformly in $t \in[0, T]$. We find that (1.3) has a unique bounded distributional solution $v$ such that $v(t, \cdot) \in \mathcal{C}_{d}^{2+\theta}\left(\mathbb{R}^{n}\right)$ for $t \in[0, T]$, and, furthermore, that there exists a positive constant $c$, independent of $g, v$ and $H$, such that

$$
\sup _{t \in[0, T]}\|v(t, \cdot)\|_{\mathcal{C}_{d}^{2+\theta}\left(\mathbb{R}^{n}\right)} \leq c\left(\|g\|_{\mathcal{C}_{d}^{2+\theta}\left(\mathbb{R}^{n}\right)}+\sup _{t \in[0, T]}\|H(t, \cdot)\|_{\mathcal{C}_{d}^{\theta}\left(\mathbb{R}^{n}\right)}\right)
$$

(see Theorem 4.3). The above mentioned metric $d$ is considered in [33, p. 11] and is related to certain distances associated to degenerate operators such as Hörmander's sum of squares of vector fields (see in particular the metric $\rho_{3}$ in $[26$, p. 112]). Furthermore, $d$ is a special case of the parabolic pseudo-metric considered in [10] (see also [18]). We refer to Section 2 for a precise definition of the metric $d$. Here we give an example of $d$. Consider the following twodimensional operator $\mathcal{A}$ :

$$
\mathcal{A} u(x, y)=\frac{1}{2} \partial_{x x}^{2} u(x, y)+F_{1}(x, y) \partial_{x} u(x, y)+(x+y) \partial_{y} u(x, y)
$$

for $(x, y) \in \mathbb{R}^{2}$, which satisfies Hypothesis 1.1 with $\tilde{p}=1$ and $k=1$. 
In this case, the metric $d$ is given by $d\left(z, z^{\prime}\right)=\left|x-x^{\prime}\right|+\left|y-y^{\prime}\right|^{1 / 3}$ for $z=(x, y), z^{\prime}=\left(x^{\prime}, y^{\prime}\right) \in \mathbb{R}^{2}$.

Let us now examine related papers on Schauder estimates. A general theory of local regularity in Sobolev and Hölder spaces is available for degenerate operators which are sums of squares of vector fields (see in particular $[12,30,16])$. Local $C^{\theta}$-estimates for operators more general than $\mathcal{A}$, in which also $q_{i j}$ are variable and time-dependent, can be found in [10] (see also [18, 25, 27] and the references therein).

Concerning global regularity results for solutions of possibly degenerate equations like (1.2) and (1.3) in spaces of continuous functions, we mention [21, 19, 20, 27, 31]. In [21] Schauder estimates are established for the Ornstein-Uhlenbeck operator $\mathcal{A}_{0}$ only assuming (1.4) and (1.5). In $[19,20]$ Schauder estimates are proved for Ornstein-Uhlenbeck type operators $\mathcal{A}_{0}$ when $F_{i}=0$ but $q_{i j}$ are not constant and can be unbounded; in $[19,20]$ it is assumed that $k \leq 1$ in hypothesis (1.5). Uniform estimates for solutions to the Cauchy problem (1.3) involving $\mathcal{A}$ with $H=0$ are given in [29]; these are obtained without any restriction on $k$ and are preliminary to the Schauder estimates of the present paper. In [31] Schauder estimates for $\mathcal{A}$ are proved assuming $k \leq 1$ in (1.5) and imposing an additional hypothesis (which is not satisfied in (1.6)).

To prove elliptic Schauder estimates, one considers the function

$$
u(x)=\int_{0}^{\infty} e^{-\lambda t} P_{t} f(x) d t, \quad x \in \mathbb{R}^{n},
$$

where $P_{t}$ is the diffusion Markov semigroup associated to $\mathcal{A}$ (i.e., $v(t, x)=$ $\left(P_{t} f\right)(x)=P_{t} f(x)$ provides the classical solution to (1.3) when $H=0$ and $g=f$; see [29]). The function $u$ is the unique bounded distributional solution to (1.2) (see Theorem 4.1). One proves global regularity properties for $u$ by means of sharp $L^{\infty}$-estimates on the spatial partial derivatives of $P_{t} f$ involving the Hölder norm of $f$ like

$$
\left\|\partial_{x_{i} x_{j} x_{r}}^{3} P_{t} f\right\|_{0} \leq c\left(\frac{1}{t^{(3-\theta) / 2}}+1\right)\|f\|_{\theta, d}, \quad t>0, i, j, r=1, \ldots, \tilde{p}
$$

(see Theorem 3.3). The behaviour in $t$ of such estimates as $t$ tends to $0^{+}$ is crucial. This is the basic idea indicated in [7] in order to study Schauder estimates for non-degenerate Kolmogorov operators. This method has been much used in recent papers also in combination with [22] (see [5, Chapter 1], $\left[3\right.$, Chapter 6] and the references therein). In [21] the $L^{\infty}$-estimates have been proved using the explicit formula for the Ornstein-Uhlenbeck semigroup $P_{t}$ associated to $\mathcal{A}_{0}$. In $[19,20,31]$ the uniform estimates are obtained by a priori estimates of Bernstein type combined with an interpolation result proved in [20, Lemma 5.1] when $k \leq 1$. Here we get the $L^{\infty}$-estimates 
involving Hölder norms by working directly on some probabilistic formulae for the spatial derivatives of $P_{t} f$ (which replace the explicit formulae used in [21]). Such formulae have been obtained in [29] using Malliavin calculus (see also $[4,17,14])$.

We believe that the probabilistic approach used here could be useful to get sharp $L^{\infty}$-estimates involving Hölder norms also in other situations. Specifically, one could consider a degenerate Kolmogorov operator like $\mathcal{A}$ in which the drift term $A x+F$ is replaced by a $C^{\infty}$-vector field $G: \mathbb{R}^{n} \rightarrow \mathbb{R}^{n}$ having additional properties. $G$ should have all derivatives bounded up to some order $k \in \mathbb{N}$ and furthermore, $e_{1}, \ldots, e_{\tilde{p}}$ and $G$ together with their commutators of length at most $k$ should span $\mathbb{R}^{n}$ at each point $x \in \mathbb{R}^{n}$. In this case global Schauder estimates are a largely open problem.

From the previous $L^{\infty}$-estimates we derive in a direct and relatively short way Schauder estimates for $u$ (see Theorem 4.2). This does not require the interpolation method of [22] which has been used in [21, 5, 20, 3, 31]. Our direct approach can be applied to prove parabolic Schauder estimates as well (see Theorem 4.3). In order to study the parabolic Cauchy problem (1.3) one proceeds initially as in the elliptic case, replacing (1.7) with the variation of constant formula (see (4.5)). However, the parabolic Schauder estimates are more involved than the corresponding elliptic ones (see Remark 3.4). In particular, they require not only the $L^{\infty}$-estimates involving Hölder norms but also the hard estimate $\left\|P_{t} g\right\|_{2+\theta, d} \leq C\|g\|_{2+\theta, d}$ for any $g \in C_{d}^{2+\theta}\left(\mathbb{R}^{n}\right)$, $t \geq 0$, where $C$ is independent of $t$ and $g$.

After some preliminaries contained in Section 2, in Section 3 we prove $L^{\infty}$-estimates for the spatial derivatives of $P_{t} f$ involving the Hölder norm of $f$. In Section 4 we show that (1.2) has a unique distributional solution and prove elliptic Schauder estimates using the results of Section 3. We also establish existence and uniqueness of space-distributional solutions to the parabolic Cauchy problem (1.3) and prove the parabolic Schauder estimates. In the final part of the paper we consider more general operators $\tilde{\mathcal{A}}$ with variable coefficients $q_{i j}(x)$. We require that the matrix $Q(x)$ has the form (1.4) where the $\tilde{p} \times \tilde{p}$ matrix $Q_{0}(x)$ is uniformly positive; furthermore, we assume that the $q_{i j}$ are $\theta$-Hölder continuous and that the $\operatorname{limit}_{x \rightarrow \infty} \lim _{0}(x)=Q_{0}^{\infty}$ exists. We obtain elliptic and parabolic Schauder estimates for $\tilde{\mathcal{A}}$, using a well-known method based on the maximum principle, a priori estimates and the continuity method (cf. [21]). Further extensions of our results are given in Remark 5.4.

We will use the letter $c$ or $C$ with subscripts for finite positive constants whose precise value is unimportant; the constants may change from proposition to proposition. 
2. Preliminaries and notation. We denote by $|\cdot|$ and $\langle\cdot, \cdot\rangle$ the Euclidean norm and the standard inner product in $\mathbb{R}^{n}$ and by $\|\cdot\|_{L}$ the operator norm in the Banach space $L\left(\mathbb{R}^{n}\right)$ of real $n \times n$ matrices. If $X$ and $Y$ are real Banach spaces, $L(X, Y)$ denotes the Banach space of all bounded and linear operators from $X$ into $Y$ endowed with the operator norm.

Let $G: \mathbb{R}^{n} \rightarrow \mathbb{R}^{m}$ be a mapping. We denote by $D G(x), D^{2} G(x)$ and $D^{3} G(x)$ respectively the first, second and third Fréchet derivative of $G$ at $x \in$ $\mathbb{R}^{n}$ when they exist (if $G$ also depends on $t$, we write $D_{x} G(t, x), D_{x x}^{2} G(t, x)$ and $\left.D_{x x x}^{3} G(t, x)\right)$.

We have $D G(x)[u], D^{2} G(x)[u][v]$ and $D^{3} G(x)[u][v][w] \in \mathbb{R}^{m}$ for $u, v, w$ $\in \mathbb{R}^{n}$. If $G$ is bounded, we set $\|G\|_{0}=\sup _{x \in \mathbb{R}^{n}}|G(x)|_{\mathbb{R}^{m}}$.

Recall that hypothesis (1.5) is known as the Kalman condition in control theory (see [35]). It is also equivalent to requiring that the symmetric matrix

$$
Q_{t}=\int_{0}^{t} e^{s A^{*}} Q e^{s A} d s
$$

is positive definite for any $t>0$ (here $e^{s A}$ denotes the exponential matrix of $A$, and $A^{*}$ the adjoint matrix of $A$ ).

As in [21] we define an orthogonal decomposition of $\mathbb{R}^{n}$ related to the Kalman condition (1.5). We consider the first $\tilde{p}$ elements $\left\{e_{1}, \ldots, e_{\tilde{p}}\right\}$ of the canonical basis in $\mathbb{R}^{n}, 1 \leq \tilde{p} \leq n$, and introduce the subspace $V_{0}=$ $\operatorname{Span}\left\{e_{1}, \ldots, e_{\tilde{p}}\right\}$.

Then set $V_{m}=\operatorname{Im}\left(Q^{1 / 2}\right)+\cdots+\operatorname{Im}\left(A^{m} Q^{1 / 2}\right)=\operatorname{Span}\left\{e_{1}, \ldots, e_{\tilde{p}}, A e_{1}, \ldots\right.$, $\left.A e_{\tilde{p}}, \ldots, A^{m} e_{1}, \ldots, A^{m} e_{\tilde{p}}\right\}$ for $1 \leq m \leq k$. One has $V_{m} \subset V_{m+1}$ and $V_{k}=\mathbb{R}^{n}$. Let $W_{0}=V_{0}, W_{1}$ be the orthogonal complement of $V_{0}$ in $V_{1}$, and $W_{m}$ be the orthogonal complement of $V_{m-1}$ in $V_{m}$ for $1 \leq m \leq k$. Defining the orthogonal projections $E_{m}$ from $\mathbb{R}^{n}$ onto $W_{m}$, one has $E_{m}\left(\mathbb{R}^{n}\right)=W_{m}$ and

$$
\mathbb{R}^{n}=\bigoplus_{m=0}^{k} E_{m}\left(\mathbb{R}^{n}\right) .
$$

We complete $\left\{e_{1}, \ldots, e_{\tilde{p}}\right\}$ to an orthonormal reference basis $\left\{e_{i}\right\}_{i=1}^{n}$ in $\mathbb{R}^{n}$ related to (2.2). It consists of generators of the subspaces and will be used throughout the paper (one can assume that $\left\{e_{i}\right\}$ is the canonical basis if $k \leq 1$, cf. $[20,31]$ ). In the following, $D_{i}, D_{i j}^{2}$, and $D_{i j r}^{3}$ will denote respectively first, second and third partial derivatives with respect to $\left\{e_{i}\right\}$. Note that if we write the operator $\mathcal{A}$ in the coordinates associated to the new basis, the second order term $\operatorname{Tr}\left(Q D^{2}\right)$ does not change. Define $I_{m}$ as the set of indices $i$ such that $e_{i}$ spans $E_{m}\left(\mathbb{R}^{n}\right), 0 \leq m \leq k$. We have

$$
I_{0}=\{1, \ldots, \tilde{p}\} .
$$


The metric $d$ associated to the operator $\mathcal{A}$ is defined using the decomposition (2.2). One first introduces the quasi-norm $\|\cdot\|,\|x\|:=\sum_{h=0}^{k}\left|E_{h} x\right|^{1 /(2 h+1)}$, $x \in \mathbb{R}^{n}$. Then we set

$$
d(x, y):=\|x-y\|=\sum_{h=0}^{k}\left|E_{h}(x-y)\right|^{1 /(2 h+1)}, \quad x, y \in \mathbb{R}^{n} .
$$

Let us introduce function spaces used to establish Schauder estimates. First we consider Euclidean function spaces and then function spaces related to the metric $d$.

We denote by $B_{b}\left(\mathbb{R}^{n}\right)$ the Banach space of all Borel and bounded functions $f: \mathbb{R}^{n} \rightarrow \mathbb{R}$, endowed with the supremum norm $\|\cdot\|_{0} ; C_{b}\left(\mathbb{R}^{n}\right)$ is the closed subspace of $B_{b}\left(\mathbb{R}^{n}\right)$ consisting of all uniformly continuous and bounded functions.

$C_{b}^{j}\left(\mathbb{R}^{n}\right), j \in \mathbb{Z}_{+}, j \geq 1$, is the Banach space of all $j$-times differentiable functions $f: \mathbb{R}^{n} \rightarrow \mathbb{R}$, whose partial derivatives, $D_{\alpha} f, \alpha \in \mathbb{Z}_{+}^{n}$, are uniformly continuous and bounded on $\mathbb{R}^{n}$ up to order $j$. This is a Banach space endowed with the norm $\|\cdot\|_{j},\|f\|_{j}=\|f\|_{0}+\sum_{|\alpha| \leq j}\left\|D_{\alpha} f\right\|_{0}, f \in C_{b}^{j}\left(\mathbb{R}^{n}\right)$. We set $C_{b}^{\infty}\left(\mathbb{R}^{n}\right)=\bigcap_{j \geq 1} C_{b}^{j}\left(\mathbb{R}^{n}\right)$. Furthermore, $C_{0}^{\infty}\left(\mathbb{R}^{n}\right)$ is the space of all functions $f \in C_{b}^{\infty}\left(\mathbb{R}^{n}\right)$ having compact support.

Fix $\theta \in(0,1)$. The space $C_{b}^{\theta}\left(\mathbb{R}^{n}\right)$ stands for the Banach space of all $\theta$-Hölder continuous and bounded functions on $\mathbb{R}^{n}$ endowed with the norm $\|f\|_{\theta}=\|f\|_{0}+[f]_{\theta}, f \in C_{b}^{\theta}\left(\mathbb{R}^{n}\right)$, where

$$
[f]_{\theta}=\sup _{z, w \in \mathbb{R}^{n}, z \neq w} \frac{|f(z)-f(w)|}{|z-w|^{\theta}}<\infty .
$$

Furthermore, $C_{b}^{2+\theta}\left(\mathbb{R}^{n}\right)=\left\{f \in C_{b}^{2}\left(\mathbb{R}^{n}\right): D_{i j}^{2} f \in C_{b}^{\theta}\left(\mathbb{R}^{n}\right), i, j=1, \ldots, n\right\}$; it is a Banach space endowed with the norm $\|f\|_{2+\theta}=\|f\|_{2}+\sum_{i, j=1}^{n}\left\|D_{i j}^{2} f\right\|_{\theta}$, $f \in C_{b}^{2+\theta}\left(\mathbb{R}^{n}\right)$. In a similar way, one defines the Banach space $C_{b}^{1+\theta}\left(\mathbb{R}^{n}\right)$.

Next, we define function spaces related to the metric $d$. Let $\gamma \in(0,3)$ be non-integer. We define $\mathcal{C}_{d}^{\gamma}\left(\mathbb{R}^{n}\right)$ as the space of all functions $f \in C_{b}\left(\mathbb{R}^{n}\right)$ such that, for any $z \in \mathbb{R}^{n}$ and for any integer $m, 0 \leq m \leq k$, the map

$$
x \mapsto f(z+x) \text { belongs to } C_{b}^{\gamma /(2 m+1)}\left(E_{m}\left(\mathbb{R}^{n}\right)\right),
$$

with $\|f(z+\cdot)\|_{\gamma /(2 m+1)}$ bounded by a constant independent of $z$ (identifying each subspace $E_{m}\left(\mathbb{R}^{n}\right)$ with $\mathbb{R}^{n(m)}$, where $n(m)=\operatorname{dim}\left[E_{m}\left(\mathbb{R}^{n}\right)\right]$, the Euclidean function spaces $C_{b}^{\gamma /(2 m+1)}\left(E_{m}\left(\mathbb{R}^{n}\right)\right)$ are well-defined $) ; \mathcal{C}_{d}^{\gamma}\left(\mathbb{R}^{n}\right)$ is a Banach space with the norm

$$
\|f\|_{\gamma, d}:=\sum_{m=0}^{k} \sup _{z \in \mathbb{R}^{n}}\|f(z+\cdot)\|_{C_{b}^{\gamma /(2 m+1)}\left(E_{m}\left(\mathbb{R}^{n}\right)\right)}, \quad f \in \mathcal{C}_{d}^{\gamma}\left(\mathbb{R}^{n}\right) .
$$


It is easy to see that if $\gamma \in(0,1)$ and $f \in C_{b}\left(\mathbb{R}^{n}\right)$, then $f \in \mathcal{C}_{d}^{\gamma}\left(\mathbb{R}^{n}\right)$ if and only if $f$ is $\gamma$-Hölder continuous with respect to the metric $d$, i.e.

$$
[f]_{\gamma, d}=\sup _{x, y \in \mathbb{R}^{n}, x \neq y}|f(x)-f(y)|\|x-y\|^{-\gamma}<\infty .
$$

Furthermore, an equivalent norm in $\mathcal{C}_{d}^{\gamma}\left(\mathbb{R}^{n}\right), \gamma \in(0,1)$, is $\|\cdot\|_{0}+[\cdot]_{\gamma, d}$. One can also define spaces $\mathcal{C}_{d}^{\alpha}\left(\mathbb{R}^{n}\right)$ for general $\alpha \in(0, \infty)$ (see [21]).

In [21, Lemma 2.1] it is proved that if $f \in \mathcal{C}_{d}^{2+\theta}\left(\mathbb{R}^{n}\right), \theta \in(0,1)$, then for any $i, j \in I_{0}$, we have both $D_{i} f \in \mathcal{C}_{d}^{\theta+1}\left(\mathbb{R}^{n}\right)$ and $D_{i j}^{2} f \in \mathcal{C}_{d}^{\theta}\left(\mathbb{R}^{n}\right)$; furthermore, there exists a constant $C$, independent of $f$, such that

$$
\left\|D_{i} f\right\|_{1+\theta, d}+\left\|D_{i j}^{2} f\right\|_{\theta, d} \leq C\|f\|_{2+\theta, d}, \quad i, j \in I_{0} .
$$

Let $f \in \mathcal{C}_{d}^{\gamma}\left(\mathbb{R}^{n}\right), \gamma \in(2,3)$. For any $x \in \mathbb{R}^{n}$, we will consider $D_{E_{0}} f(x) \in \mathbb{R}^{n}$, the gradient of $f$ at $x \in \mathbb{R}^{n}$ in the directions of $E_{0}\left(\mathbb{R}^{n}\right)$, i.e.,

$$
D_{E_{0}} f(x)=\left(D_{1} f(x), \ldots, D_{\tilde{p}} f(x), 0, \ldots, 0\right)
$$

and, similarly, the $n \times n$ Hessian matrix $D_{E_{0}}^{2} f(x)$ in the directions of $E_{0}\left(\mathbb{R}^{n}\right)$, i.e., $\left(D_{E_{0}}^{2} f(x)\right)_{i j}=D_{i j}^{2} f(x)$ if $i, j \in I_{0}$, and $\left(D_{E_{0}}^{2} f(x)\right)_{i j}=0$ otherwise.

We finish the section with an equivalent definition of $\mathcal{C}_{d}^{\gamma}\left(\mathbb{R}^{n}\right)$ involving the quasi-norm $\|\cdot\|$. Let $f \in C_{b}\left(\mathbb{R}^{n}\right)$. We introduce, for any $x, v \in \mathbb{R}^{n}$,

$$
\triangle_{v}^{3} f(x)=f(x)-3 f(x+v)+3 f(x+2 v)-f(x+3 v) .
$$

Lemma 2.1. Let $\gamma \in(0,3)$ be non-integer. Let $f \in C_{b}\left(\mathbb{R}^{n}\right)$. Then $f \in$ $\mathcal{C}_{d}^{\gamma}\left(\mathbb{R}^{n}\right)$ if and only if

$$
[f]_{\gamma, d, 3}=\sup _{x, v \in \mathbb{R}^{n}, v \neq 0,\|v\| \leq 1}\left|\triangle_{v}^{3} f(x)\right|\|v\|^{-\gamma}<\infty .
$$

Furthermore, $\|\cdot\|_{0}+[\cdot]_{\gamma, d, 3}$ is equivalent to $\|\cdot\|_{\gamma, d}$.

Proof. We use the following result of Triebel (see [34, Section 2.7.2]). Let $g \in C_{b}\left(\mathbb{R}^{n}\right)$. Then $g$ belongs to $C_{b}^{\gamma}\left(\mathbb{R}^{n}\right), \gamma \in(0,3)$ non-integer, if and only if

$$
[g]_{\gamma, 3}=\sup _{x \in \mathbb{R}^{n},|v| \leq 1, v \neq 0}|v|^{-\gamma}\left|\triangle_{v}^{3} g(x)\right|<\infty .
$$

Furthermore, in $C_{b}^{\gamma}\left(\mathbb{R}^{n}\right)$ the norm $\|\cdot\|_{\gamma}$ is equivalent to $\|\cdot\|_{0}+[\cdot]_{\gamma, 3}$.

$\Rightarrow$ Let $f \in \mathcal{C}_{d}^{\gamma}\left(\mathbb{R}^{n}\right)$ and fix $v \in \mathbb{R}^{n}$. We set $v=v_{0}+v_{1}$, where $v_{0}=E_{0} v$ and $v_{1}=\sum_{h=1}^{k} E_{h} v=v-E_{0} v$ (see (2.2)). We get, for any $x \in \mathbb{R}^{n}$,

$$
\begin{aligned}
& \left|\triangle_{v}^{3} f(x)\right| \leq\left|f(x)-f\left(x+v_{1}\right)\right| \\
& \quad+\left|f\left(x+v_{1}\right)-3 f\left(x+v_{1}+v_{0}\right)+3 f\left(x+v_{1}+2 v_{0}\right)-f\left(x+v_{1}+3 v_{0}\right)\right| \\
& \quad+3\left|f\left(x+2 v_{1}+2 v_{0}\right)-f\left(x+v_{1}+2 v_{0}\right)\right|
\end{aligned}
$$




$$
\begin{aligned}
& +\left|f\left(x+v_{1}+3 v_{0}\right)-f\left(x+3 v_{1}+3 v_{0}\right)\right| \\
\leq & \|f\|_{\gamma, d}\left(4 \sum_{h=1}^{k}\left|E_{h} v\right|^{\gamma /(2 h+1)}+\sum_{h=1}^{k}\left|E_{h}(2 v)\right|^{\gamma /(2 h+1)}+\left|v_{0}\right|^{\gamma}\right) \\
\leq & C\|f\|_{\gamma, d}\|v\|^{\gamma} .
\end{aligned}
$$

$\Leftarrow$ Let $f \in C_{b}\left(\mathbb{R}^{n}\right)$ and take $v_{h} \in E_{h}\left(\mathbb{R}^{n}\right)$ with $0 \leq h \leq k$. By assumption, we know that $\left|\triangle_{v_{h}}^{3} f(x)\right| \leq[f]_{\gamma, d, 3}\left|v_{h}\right|^{\gamma /(2 h+1)}$ for any $x \in \mathbb{R}^{n}$. It follows that $f(x+\cdot) \in C_{b}^{\gamma /(2 h+1)}\left(E_{h}\left(\mathbb{R}^{n}\right)\right)$ and there exists $C>0$ independent of $f$ and $x$ such that $\|f(x+\cdot)\|_{C_{b}^{\gamma /(2 h+1)}\left(E_{h}\left(\mathbb{R}^{n}\right)\right)} \leq C\left(\|f\|_{0}+[f]_{\gamma, d, 3}\right), 0 \leq h \leq k$. Thus $f \in \mathcal{C}_{d}^{\gamma}\left(\mathbb{R}^{n}\right)$.

3. Estimates on the diffusion semigroup associated to $\mathcal{A}$. In this section we consider the diffusion semigroup $P_{t}$ associated to the operator $\mathcal{A}$ (i.e., $v(t, x)=\left(P_{t} g\right)(x)=P_{t} g(x)$ provides the classical solution to (1.3) when $H=0$ and $g$ is smooth enough). We obtain $L^{\infty}$-estimates on the first, second and third spatial partial derivatives of $P_{t} f$ in terms of the Hölder norm of $f$. These estimates will lead in the next section to Schauder estimates for (1.2) and (1.3).

Let $\left(\Omega,\left(\mathcal{F}_{t}\right)_{t \geq 0}, \mathcal{F}, \mathbb{P}\right)$ be a complete stochastic basis (satisfying the usual assumptions; see, for instance, [24]). Let $W_{t}, t \geq 0$, be a standard $n$ dimensional Wiener process defined and adapted on the stochastic basis. Let $X_{t}^{x}$ be the unique (strong) solution to the SDE

$$
X_{t}^{x}=x+\int_{0}^{t} A X_{s}^{x} d s+\int_{0}^{t} F\left(X_{s}^{x}\right) d s+Q^{1 / 2} W_{t}, \quad t \geq 0, x \in \mathbb{R}^{n},
$$

$\mathbb{P}$-a.s., where the matrix $A$ is the same as in (1.1) and $Q^{1 / 2}$ is the unique $n \times n$ symmetric non-negative definite square root of $Q$. The diffusion semigroup $P_{t}$ associated to $\mathcal{A}$ is the family of linear contractions $P_{t}: B_{b}\left(\mathbb{R}^{n}\right) \rightarrow B_{b}\left(\mathbb{R}^{n}\right)$, $t \geq 0$, defined by

$$
P_{t} g(x):=\mathbb{E}\left[g\left(X_{t}^{x}\right)\right], \quad t \geq 0, g \in B_{b}\left(\mathbb{R}^{n}\right), x \in \mathbb{R}^{n},
$$

where the expectation is taken with respect to $\mathbb{P}$. Introducing the OrnsteinUhlenbeck process $Z_{t}^{x}$ which solves (3.1) when $F=0$, i.e.,

$$
Z_{t}^{x}=e^{t A} x+Z_{t}^{0}, \quad \text { where } \quad Z_{t}^{0}=\int_{0}^{t} e^{(t-s) A} Q^{1 / 2} d W_{s}
$$

we have $X_{t}^{x}=Z_{t}^{x}+\int_{0}^{t} e^{(t-s) A} F\left(X_{s}^{x}\right) d s$.

Let us recall an application of the Girsanov theorem which will be used in the proof of Theorem 3.3 (see also [29]). Fix $t>0$ and $x \in \mathbb{R}^{n}$, and define 
$Q^{-1 / 2}=\left(\begin{array}{cc}Q_{0}^{-1 / 2} & 0 \\ 0 & 0\end{array}\right) ;$ then consider the stochastic process

$$
L_{s}^{x}:=W_{s}-\int_{0}^{s}\left(Q^{-1 / 2} F\right)\left(Z_{r}^{x}\right) d r=W_{s}-\int_{0}^{s} G\left(Z_{r}^{x}\right) d r, \quad s \in[0, t],
$$

where we have set $G:=Q^{-1 / 2} F$. By the Girsanov theorem, the process $L_{s}^{x}$ is a Wiener process on $\left(\Omega,\left(\mathcal{F}_{s}\right)_{s \leq t}, \mathcal{F}_{t}, \mathbb{Q}\right)$, where $\mathbb{Q}$ is a probability measure on $\left(\Omega, \mathcal{F}_{t}\right)$ having density $\Phi(t, x)$ with respect to $\mathbb{P}$, i.e.,

$$
\mathbb{Q}(A):=\mathbb{E}\left[1_{A} \Phi(t, x)\right]
$$

for any $A \in \mathcal{F}_{t}$, where

$$
\Phi(t, x)=\exp \left(\int_{0}^{t}\left\langle G\left(Z_{s}^{x}\right), d W_{s}\right\rangle-\frac{1}{2} \int_{0}^{t}\left|G\left(Z_{s}^{x}\right)\right|^{2} d s\right) .
$$

The processes $Z^{x}=\left(Z_{s}^{x}\right)$ and $X^{x}=\left(X_{s}^{x}\right), s \in[0, t]$, satisfy the same equation $(3.1)$ in $\left(\Omega, \mathcal{F}_{t}, \mathbb{Q},\left(L_{s}^{x}\right)\right)$ and $\left(\Omega, \mathcal{F}_{t}, \mathbb{P},\left(W_{s}\right)\right)$ respectively. Therefore, by uniqueness, their laws on $C\left([0, t] ; \mathbb{R}^{n}\right)$ are the same (under the probability measures $\mathbb{Q}$ and $\mathbb{P}$ respectively). This implies that

$$
P_{t} f(x)=\mathbb{E}\left[f\left(X_{t}^{x}\right)\right]=\mathbb{E}\left[f\left(Z_{t}^{x}\right) \Phi(t, x)\right], \quad f \in B_{b}\left(\mathbb{R}^{n}\right) .
$$

The next theorem is proved in [29]. It provides probabilistic formulae and preliminary uniform estimates for the spatial partial derivatives of $P_{t} f$ up to the third order (the formula for the first derivatives was obtained in [14]). The proof uses Malliavin calculus. Related formulae for the spatial derivatives of degenerate diffusion semigroups are given in $[4,17]$.

TheOREM 3.1. Under Hypothesis 1.1, the following statements hold:

(i) For any $t>0$ and $f \in B_{b}\left(\mathbb{R}^{n}\right)$, the function $P_{t} f(\cdot)$ is three times differentiable on $\mathbb{R}^{n}$ with all derivatives bounded up to the third order.

(ii) There exist random variables $J_{i}^{1}(t, x), J_{i j}^{2}(t, x)$ and $J_{i j r}^{3}(t, x), t>0$, $x \in \mathbb{R}^{n}, i, j, r \in\{1, \ldots, n\}$, which belong to $L^{q}(\Omega)$, for any $q \geq 1$, and such that

$$
\begin{aligned}
D_{i}\left(P_{t} g\right)(x) & =D_{i} P_{t} g(x)=\mathbb{E}\left[g\left(X_{t}^{x}\right) J_{i}^{1}(t, x)\right], \\
D_{i j}^{2} P_{t} g(x) & =\mathbb{E}\left[g\left(X_{t}^{x}\right) J_{i j}^{2}(t, x)\right], \\
D_{i j r}^{3} P_{t} g(x) & =\mathbb{E}\left[g\left(X_{t}^{x}\right) J_{i j r}^{3}(t, x)\right], \quad g \in C_{b}\left(\mathbb{R}^{n}\right) .
\end{aligned}
$$

(iii) For any $t>0, q \geq 1, x \in \mathbb{R}^{n}$, we have the following estimates:
(a) $\mathbb{E}\left|J_{i}^{1}(t, x)\right|^{q} \leq c_{q}(t)\left|Q_{t}^{-1 / 2} e^{t A} e_{i}\right|^{q} ;$
(b) $\mathbb{E}\left|J_{i j}^{2}(t, x)\right|^{q} \leq c_{q}(t)\left|Q_{t}^{-1 / 2} e^{t A} e_{i}\right|^{q}\left|Q_{t}^{-1 / 2} e^{t A} e_{j}\right|^{q}$;
(c) $\mathbb{E}\left|J_{i j r}^{3}(t, x)\right|^{q} \leq c_{q}(t)\left|Q_{t}^{-1 / 2} e^{t A} e_{i}\right|^{q}\left|Q_{t}^{-1 / 2} e^{t A} e_{j}\right|^{q}\left|Q_{t}^{-1 / 2} e^{t A} e_{r}\right|^{q}$, 
where $c_{q}(t)$ is a continuous and increasing function on $[0, \infty)$, and $c_{q}(t)=$ $c\left(q, t,\|D F\|_{0},\left\|D^{2} F\right\|_{0},\left\|D^{3} F\right\|_{0}, \tilde{p}, \nu_{1}, A, n\right)$, where the integer $\tilde{p}$ is introduced in (1.4).

It is worth noticing that the quantity $\left|Q_{t}^{-1 / 2} e^{t A} e_{i}\right|^{2}$, corresponding to $q=2$, has a well-known control-theoretic interpretation; see, for instance, [35].

Furthermore, the following estimates are known (see [32] and [21, (3.4)]):

$$
\left|Q_{t}^{-1 / 2} e^{t A} e_{i}\right| \leq \frac{c}{t^{h+1 / 2}}, \quad e_{i} \in E_{h}\left(\mathbb{R}^{n}\right), 0 \leq h \leq k, t \in(0,1] .
$$

where $c=c\left(\tilde{p}, \nu_{1}, \nu_{2}, A, n\right)>0$ and the integer $k$ is defined in (1.5). Estimates (3.8) can also be deduced by purely control-theoretic arguments, using [35, Proposition I.1.3] together with [2].

COROllary 3.2. There exists $c=c\left(\tilde{p}, \nu_{1}, \nu_{2}, A, n,\|D F\|_{0},\left\|D^{2} F\right\|_{0}\right.$, $\left.\left\|D^{3} F\right\|_{0}\right)>0$ such that the following estimates hold, for any $t>0, g \in$ $B_{b}\left(\mathbb{R}^{n}\right)$, and indices $i \in I_{h}, j \in I_{h^{\prime}}$ and $r \in I_{h^{\prime \prime}}$, where $h, h^{\prime}, h^{\prime \prime} \in\{0, \ldots, k\}$ :

$$
\begin{aligned}
\left\|D_{i} P_{t} g\right\|_{0} & \leq c\left(\frac{1}{t^{h+1 / 2}}+1\right)\|g\|_{0}, \\
\left\|D_{i j}^{2} P_{t} g\right\|_{0} & \leq c\left(\frac{1}{t^{h+h^{\prime}+1}}+1\right)\|g\|_{0}, \\
\left\|D_{i j r}^{3} P_{t} g\right\|_{0} & \leq c\left(\frac{1}{t^{h+h^{\prime}+h^{\prime \prime}+3 / 2}}+1\right)\|g\|_{0} .
\end{aligned}
$$

Proof. It is enough to prove the estimates when $g \in C_{b}\left(\mathbb{R}^{n}\right)$ (see, for instance, [29, Remark 3.5]). Using Theorem 3.1 and (3.8), we first establish the estimates assuming in addition that $0<t<1$. We have, for any $x \in$ $\mathbb{R}^{n}, t \in(0,1)$,

$$
\left|D_{i} P_{t} g(x)\right| \leq\|g\|_{0} \mathbb{E}\left|J_{i}^{1}(t, x)\right| \leq c_{1}\left|Q_{t}^{-1 / 2} e^{t A} e_{i}\right|\|g\|_{0} \leq \frac{c}{t^{h+1 / 2}}\|g\|_{0} .
$$

In a similar way, we get the second and third estimates for $t<1$.

When $t \geq 1$, by the semigroup and contraction properties of $P_{t}$, we have

$$
\left\|D_{i} P_{t} g\right\|_{0}=\left\|D_{i} P_{1 / 2}\left(P_{t-1 / 2} g\right)\right\|_{0} \leq c 2^{h+1 / 2}\left\|P_{(2 t-1) / 2} g\right\|_{0} \leq c 2^{h+1 / 2}\|g\|_{0}
$$

for any $i \in I_{h}$. Thus the required estimate of $D_{i} P_{t} g$ is proved for any $t>0$. Similarly, we get the other estimates for any $t>0$.

The main result of the section is the following theorem. Its proof also allows one to complete the final part of the proof of [21, Theorem 3.4]. We set $a \wedge b=\min (a, b)$.

TheOREM 3.3. Fix any non-integer $\gamma \in(0,3)$. There exists $c=c\left(\gamma, \tilde{p}, \nu_{1}\right.$, $\left.\nu_{2}, A, n,\|D F\|_{0},\left\|D^{2} F\right\|_{0},\left\|D^{3} F\right\|_{0}\right)>0$ such that, for any $f \in \mathcal{C}_{d}^{\gamma}\left(\mathbb{R}^{n}\right), t>0$, 
and any indices $i \in I_{h}, j \in I_{h^{\prime}}$ and $r \in I_{h^{\prime \prime}}$, where $h, h^{\prime}, h^{\prime \prime} \in\{0, \ldots, k\}$,

$$
\begin{aligned}
& \text { (i) }\left\|D_{i} P_{t} f\right\|_{0} \leq c\left(\frac{1}{(t \wedge 1)^{(1-\gamma) / 2+h}}+1\right)\|f\|_{\gamma, d}, \\
& \text { (ii) }\left\|D_{i j}^{2} P_{t} f\right\|_{0} \leq c\left(\frac{1}{(t \wedge 1)^{h+h^{\prime}+(2-\gamma) / 2}}+1\right)\|f\|_{\gamma, d}, \\
& \text { (iii) }\left\|D_{i j r}^{3} P_{t} f\right\|_{0} \leq c\left(\frac{1}{t^{h+h^{\prime}+h^{\prime \prime}+(3-\gamma) / 2}}+1\right)\|f\|_{\gamma, d}, \\
& \text { (iv) }\left\|P_{t} f\right\|_{\gamma, d} \leq c\|f\|_{\gamma, d} .
\end{aligned}
$$

REMARK 3.4. Estimates (i)-(iv) will be used to get elliptic and parabolic Schauder estimates for $\mathcal{A}$. However, we stress that to prove elliptic Schauder estimates we only need a special case of (3.10). More precisely, we need the following estimates, for any $\theta \in(0,1), f \in \mathcal{C}_{d}^{\theta}\left(\mathbb{R}^{n}\right), t>0$ and any indices $i, j \in I_{0}, r \in I_{h}$, with $h \in\{0, \ldots, k\}$ :

$$
\begin{aligned}
& \text { (a) }\left\|D_{r} P_{t} f\right\|_{0} \leq c\left(\frac{1}{t^{(1-\theta) / 2+h}}+1\right)\|f\|_{\theta, d}, \\
& \text { (b) }\left\|D_{i j}^{2} P_{t} f\right\|_{0} \leq c\left(\frac{1}{t^{(2-\theta) / 2}}+1\right)\|f\|_{\theta, d}, \\
& \text { (c) }\left\|D_{i j r}^{3} P_{t} f\right\|_{0} \leq c\left(\frac{1}{t^{(3-\theta) / 2+h}}+1\right)\|f\|_{\theta, d}, \\
& \text { (d) }\left\|P_{t} f\right\|_{\theta, d} \leq c\|f\|_{\theta, d} .
\end{aligned}
$$

These estimates are simpler to obtain than the general ones in which $\gamma \in$ $(0,3)$. On the other hand, note that the estimates (3.10)(iv) with $\gamma \in(2,3)$ are a particular case of the parabolic Schauder estimates corresponding to $H=0$ in (1.3) (see Theorem 4.3). Estimates (iv) with $\gamma \in(2,3)$ will be deduced from (iii).

In order to prove the main result we need three preliminary lemmas. To state the first one, we introduce the deterministic process

$$
Y_{t}^{x}=e^{t A} x+\int_{0}^{t} e^{(t-s) A} F\left(Y_{s}^{x}\right) d s, \quad t \geq 0, x \in \mathbb{R}^{n},
$$

which solves

$$
\left\{\begin{array}{l}
\dot{Y}_{t}=A Y_{t}+F\left(Y_{t}\right) \\
Y_{0}=x
\end{array}\right.
$$

Lemma 3.5. For any $q>0$, there exists $C=C\left(q, \tilde{p}, \nu_{1}, \nu_{2}, n, A,\|D F\|_{0}\right)$ $>0$ such that

$$
\sup _{x \in \mathbb{R}^{n}} \mathbb{E}\left[\left(d\left(X_{t}^{x}, Y_{t}^{x}\right)\right)^{q}\right]=\sup _{x \in \mathbb{R}^{n}} \mathbb{E}\left\|X_{t}^{x}-Y_{t}^{x}\right\|^{q} \leq C t^{q / 2}, \quad 0 \leq t \leq 1 .
$$


Proof. Note that (3.13) is equivalent to the following assertion: for any $q>0$ and $0 \leq h \leq k$, there exists $C_{1}>0$ such that

$$
\sup _{x \in \mathbb{R}^{n}} \mathbb{E}\left|E_{h}\left(X_{t}^{x}-Y_{t}^{x}\right)\right|^{q} \leq C_{1} t^{q(2 h+1) / 2}, \quad 0 \leq t \leq 1
$$

(see (2.3)). Let us prove (3.14). Since there exists $c>0$ such that $|x| \leq$ $c \sum_{h=0}^{k}\left|E_{h} x\right|$ for any $x \in \mathbb{R}^{n}$, we get

$$
\begin{aligned}
\left|E_{h}\left(X_{t}^{x}-Y_{t}^{x}\right)\right| & \leq\left|\int_{0}^{t}\left(E_{h} e^{(t-r) A} E_{0}\right)\left[F\left(X_{r}^{x}\right)-F\left(Y_{r}^{x}\right)\right] d r\right|+\left|E_{h} Z_{t}^{0}\right| \\
& \leq c\|D F\|_{0} \sum_{j=0}^{k} \int_{0}^{t}\left\|E_{h} e^{(t-r) A} E_{0}\right\|_{L}\left|E_{j}\left(X_{r}^{x}-Y_{r}^{x}\right)\right| d r+\left|E_{h} Z_{t}^{0}\right|
\end{aligned}
$$

$\mathbb{P}$-a.s. Using the estimate (see [21, Lemma 3.1])

$$
\left\|E_{h} e^{s A} E_{0}\right\|_{L} \leq c^{\prime \prime} s^{h}, \quad s \in[0,1], 0 \leq h \leq k, c^{\prime \prime}=c^{\prime \prime}(A)>0,
$$

we arrive at

$$
\begin{array}{r}
\left|E_{h}\left(X_{t}^{x}-Y_{t}^{x}\right)\right| \leq\left|E_{h} Z_{t}^{0}\right|+C \int_{0}^{t}(t-r)^{h}\left|E_{h}\left(X_{r}^{x}-Y_{r}^{x}\right)\right| d r \\
+C \sum_{j=0, j \neq h}^{k} \int_{0}^{t}(t-r)^{h}\left|E_{j}\left(X_{r}^{x}-Y_{r}^{x}\right)\right| d r
\end{array}
$$

$\mathbb{P}$-a.s. Now we use the fact that $\left|E_{j}\left(X_{r}^{x}-Y_{r}^{x}\right)\right| \leq\left|X_{r}^{x}-Y_{r}^{x}\right|$ for any $0 \leq j \leq k$.

In order to estimate $\left|X_{r}^{x}-Y_{r}^{x}\right|$, note that

$$
\left|X_{t}^{x}-Y_{t}^{x}\right| \leq C^{\prime} \int_{0}^{t}\left|X_{s}^{x}-Y_{s}^{x}\right| d s+\left|Z_{t}^{0}\right| .
$$

An application of the Gronwall lemma gives, $\mathbb{P}$-a.s.,

$$
\left|X_{t}^{x}-Y_{t}^{x}\right| \leq\left|Z_{t}^{0}\right|+C^{\prime} \int_{0}^{t}\left|Z_{s}^{0}\right| e^{(t-s) C^{\prime}} d s \leq\left|Z_{t}^{0}\right|+C_{1} \int_{0}^{t}\left|Z_{s}^{0}\right| d s
$$

for $0 \leq t \leq 1$. Using estimate (3.17) in (3.16) we get

$$
\begin{aligned}
\left|E_{h}\left(X_{t}^{x}-Y_{t}^{x}\right)\right| \leq & \left|E_{h} Z_{t}^{0}\right|+C \int_{0}^{t}(t-r)^{h}\left|E_{h}\left(X_{r}^{x}-Y_{r}^{x}\right)\right| d r \\
& +C k \int_{0}^{t}(t-r)^{h}\left[\left|Z_{r}^{0}\right|+C_{1} \int_{0}^{r}\left|Z_{s}^{0}\right| d s\right] d r
\end{aligned}
$$

$\mathbb{P}$-a.s. Let now $q \in \mathbb{Z}_{+}$and recall that $0 \leq t \leq 1$. We have 


$$
\begin{aligned}
\left|E_{h}\left(X_{t}^{x}-Y_{t}^{x}\right)\right|^{q} \leq & C_{3}\left(\left|E_{h} Z_{t}^{0}\right|^{q}+\int_{0}^{t}\left|E_{h}\left(X_{r}^{x}-Y_{r}^{x}\right)\right|^{q} d r\right. \\
& \left.+\int_{0}^{t}(t-r)^{h q}\left[\left|Z_{r}^{0}\right|^{q}+\int_{0}^{r}\left|Z_{s}^{0}\right|^{q} d s\right] d r\right)
\end{aligned}
$$

$\mathbb{P}$-a.s. Before applying the expectation in the last formula, we check that

$$
\mathbb{E}\left|E_{h} Z_{t}^{0}\right|^{q}=\mathbb{E}\left|\int_{0}^{t} E_{h} e^{(t-s) A} Q^{1 / 2} d W_{s}\right|^{q} \leq c_{q, h} t^{q(2 h+1) / 2}
$$

for $0 \leq t \leq 1, q>0$, and $0 \leq h \leq k$, where $E_{h}$ are the orthogonal projections introduced in (2.2). Denoting by $N\left(0, Q_{t}\right)$ the Gaussian measure on $\mathbb{R}^{n}$ with mean 0 and covariance matrix $Q_{t}$ given in (2.1), we have

$$
\begin{aligned}
\mathbb{E}\left|E_{h} Z_{t}^{0}\right|^{q} & =\int_{\mathbb{R}^{n}}\left|E_{h} y\right|^{q} N\left(0, Q_{t}\right) d y=\int_{\mathbb{R}^{n}}\left|E_{h} Q_{t}^{1 / 2} z\right|^{q} N(0, I) d z \\
& \leq\left\|E_{h} Q_{t}^{1 / 2}\right\|_{L}^{q} \int_{\mathbb{R}^{n}}|z|^{q} N(0, I) d z \leq c t^{q(2 h+1) / 2}
\end{aligned}
$$

for $t \leq 1$, where $I$ is the $n \times n$ identity matrix. In the last inequality we have used the fact that $\left\|E_{h} Q_{t}^{1 / 2}\right\|_{L} \leq c^{\prime} t^{(2 h+1) / 2}$ for $0 \leq t \leq 1$ and $0 \leq h \leq k$, where $c^{\prime}=c^{\prime}\left(\tilde{p}, n, A, \nu_{1}, \nu_{2}\right)$ (see $\left.[21,(3.2)]\right)$.

By (3.18), we infer

$$
\begin{aligned}
& \mathbb{E}\left|E_{h}\left(X_{t}^{x}-Y_{t}^{x}\right)\right|^{q} \leq C_{3}\left(\mathbb{E}\left|E_{h} Z_{t}^{0}\right|^{q}+\right. \int_{0}^{t} \mathbb{E}\left|E_{h}\left(X_{r}^{x}-Y_{r}^{x}\right)\right|^{q} d r \\
&\left.+\int_{0}^{t}(t-r)^{h q}\left[\mathbb{E}\left|Z_{r}^{0}\right|^{q}+\int_{0}^{r} \mathbb{E}\left|Z_{s}^{0}\right|^{q} d s\right] d r\right) \\
& \leq C_{4}\left(t^{q(2 h+1) / 2}+\int_{0}^{t} \mathbb{E}\left|E_{h}\left(X_{r}^{x}-Y_{r}^{x}\right)\right|^{q} d r+\int_{0}^{t}(t-r)^{h q}\left[r^{q / 2}+\frac{r^{1+q / 2}}{1+q / 2}\right] d r\right) .
\end{aligned}
$$

Since

$$
\int_{0}^{t}(t-s)^{p} s^{r} d s=\frac{p !}{(r+p+1)(r+p) \ldots(r+1)} t^{r+p+1}, \quad p \in \mathbb{Z}_{+}, r>0,
$$

we find

$$
\begin{aligned}
& \mathbb{E}\left|E_{h}\left(X_{t}^{x}-Y_{t}^{x}\right)\right|^{q} \\
& \quad \leq C_{5}\left(t^{q(2 h+1) / 2}+\int_{0}^{t} \mathbb{E}\left|E_{h}\left(X_{r}^{x}-Y_{r}^{x}\right)\right|^{q} d r+2 \int_{0}^{t}(t-r)^{h q} r^{q / 2} d r\right)
\end{aligned}
$$




$$
\begin{aligned}
& \leq C_{6}\left(t^{q(2 h+1) / 2}+\int_{0}^{t} \mathbb{E}\left|E_{h}\left(X_{r}^{x}-Y_{r}^{x}\right)\right|^{q} d r+t^{h q+1+q / 2}\right) \\
& \leq 2 C_{6}\left(t^{q(2 h+1) / 2}+\int_{0}^{t} \mathbb{E}\left|E_{h}\left(X_{r}^{x}-Y_{r}^{x}\right)\right|^{q} d r\right), \quad t \leq 1 .
\end{aligned}
$$

Applying the Gronwall lemma, we get

$$
\mathbb{E}\left|E_{h}\left(X_{t}^{x}-Y_{t}^{x}\right)\right|^{q} \leq C_{7} t^{q(2 h+1) / 2}, \quad 0 \leq t \leq 1 .
$$

Now if $q>0$, we consider an integer $m \geq q$. By the Jensen inequality,

$$
\left(\mathbb{E}\left|E_{h}\left(X_{t}^{x}-Y_{t}^{x}\right)\right|^{q}\right)^{m / q} \leq \mathbb{E}\left|E_{h}\left(X_{t}^{x}-Y_{t}^{x}\right)\right|^{m} \leq C t^{m(2 h+1) / 2}, \quad t \leq 1 .
$$

This implies that $\mathbb{E}\left|E_{h}\left(X_{t}^{x}-Y_{t}^{x}\right)\right|^{q} \leq C^{q / m} t^{q(2 h+1) / 2}$. The assertion is proved.

Lemma 3.6. There exists $\Omega_{0} \in \mathcal{F}$ with $\mathbb{P}\left(\Omega_{0}\right)=1$ such that for any $\omega \in \Omega_{0}$ and $t \in[0,1]$, the mapping $x \mapsto X_{t}^{x}(\omega) \in \mathbb{R}^{n}$ is differentiable up to the third order on $\mathbb{R}^{n}$. Furthermore, for any $i, j, r \in\{0, \ldots, n\}$ and $x \in \mathbb{R}^{n}$, there exist continuous adapted stochastic processes $\left(\eta_{i}(t, x)\right),\left(\eta_{i j}(t, x)\right)$ and $\left(\eta_{i j r}(t, x)\right)$ with values in $\mathbb{R}^{n}$ and $C=C\left(\|D F\|_{0},\left\|D^{2} F\right\|_{0},\left\|D^{3} F\right\|_{0},\|A\|_{L}\right)>$ 0 such that

$$
\begin{aligned}
& \eta_{i}(t, x)=D_{i} X_{t}^{x}=\lim _{h \rightarrow 0}\left(X_{t}^{x+h e_{i}}-X_{t}^{x}\right) h^{-1}, \\
& \eta_{i j}(t, x)=D_{i j}^{2} X_{t}^{x}, \quad \eta_{i j r}(t, x)=D_{i j r}^{3} X_{t}^{x}, \\
& \left|\eta_{i}(t, x)\right|+\left|\eta_{i j}(t, x)\right|+\left|\eta_{i j r}(t, x)\right| \leq C,
\end{aligned}
$$

$\mathbb{P}$-a.s. for any $t \in[0,1]$ and $x \in \mathbb{R}^{n}$.

Proof. The proof is straightforward. We include it for the sake of completeness. For $\omega \in \Omega, \mathbb{P}$-a.s., introduce the Banach space $E=C\left([0,1] ; \mathbb{R}^{n}\right)$. Define the map $\mathcal{F}: \mathbb{R}^{n} \times E \rightarrow E$ by

$$
\mathcal{F}(x, u)(t):=u(t)-x-\int_{0}^{t}(A u(r)+F(u(r))) d r-\sqrt{Q} W_{t}(\omega)
$$

for $t \in[0,1], u \in E$, and $x \in \mathbb{R}^{n}$. Applying the implicit function theorem, we find that the mapping $x \mapsto X_{(\cdot)}^{x}(\omega)$ from $\mathbb{R}^{n}$ into $E$ is three times Fréchet differentiable. Denote by $\eta_{i}(t, x), \eta_{i j}(t, x)$ and $\eta_{i j r}(t, x) t \in[0,1]$, respectively the first (directional) derivative at $x \in \mathbb{R}^{n}$ in the direction $e_{i}$, the second derivative at $x$ in the directions $e_{i}$ and $e_{j}$, and the third derivative at $x$ in the directions $e_{i}, e_{j}$ and $e_{r}$, where $i, j, r=1, \ldots, n$. Note that $\eta_{i}(t, x), \eta_{i j}(t, x)$ and $\eta_{i j r}(t, x)$ solve, $\mathbb{P}$-a.s., the variation equations 


$$
\begin{aligned}
\eta_{i}(t, x)= & e_{i}+\int_{0}^{t}\left(A \eta_{i}(s, x)+D F\left(X_{s}^{x}\right)\left[\eta_{i}(s, x)\right]\right) d s \\
\eta_{i j}(t, x)= & \int_{0}^{t}\left(A \eta_{i j}(s, x)\right. \\
& \left.+D^{2} F\left(X_{s}^{x}\right)\left[\eta_{i}(s, x)\right]\left[\eta_{j}(s, x)\right]+D F\left(X_{s}^{x}\right)\left[\eta_{i j}(s, x)\right]\right) d s \\
& t \\
\eta_{i j r}(t, x)= & \int_{0}^{t}\left(A \eta_{i j r}(s, x)+D^{3} F\left(X_{s}^{x}\right)\left[\eta_{i}(s, x)\right]\left[\eta_{j}(s, x)\right]\left[\eta_{r}(s, x)\right]\right) d s \\
& +\int_{0}^{t}\left(D^{2} F\left(X_{s}^{x}\right)\left[\eta_{i r}(s, x)\right]\left[\eta_{j}(s, x)\right]\right. \\
& \left.+D^{2} F\left(X_{s}^{x}\right)\left[\eta_{i}(s, x)\right]\left[\eta_{j r}(s, x)\right]+D F\left(X_{s}^{x}\right)\left[\eta_{i j r}(s, x)\right]\right) d s,
\end{aligned}
$$

for $t \in[0,1]$. It follows easily that $\left(\eta_{i}(\cdot, x)\right),\left(\eta_{i j}(\cdot, x)\right)$ and $\left(\eta_{i j r}(\cdot, x)\right)$ are continuous adapted stochastic processes. An application of the Gronwall lemma gives the final assertion.

LEMMA 3.7. Let $f \in \mathcal{C}_{d}^{\gamma}\left(\mathbb{R}^{n}\right), \gamma \in(2,3)$, and $i, j, r \in\{1, \ldots, n\}$. Consider the following random variables depending on $t \in(0,1)$ and $x \in \mathbb{R}^{n}$ (see (2.6) and (3.6)):

$$
\begin{aligned}
\Lambda(t, x)= & \left\langle D_{E_{0}} f\left(Y_{t}^{x}\right), E_{0}\left(X_{t}^{x}-Y_{t}^{x}\right)\right\rangle \\
& +\frac{1}{2}\left\langle D_{E_{0}}^{2} f\left(Y_{t}^{x}\right)\left[E_{0}\left(X_{t}^{x}-Y_{t}^{x}\right)\right], E_{0}\left(X_{t}^{x}-Y_{t}^{x}\right)\right\rangle .
\end{aligned}
$$

The functions $\phi_{i}(x, t)=\mathbb{E}\left[\Lambda(t, x) J_{i}^{1}(t, x)\right], \phi_{i j}(x, t)=\mathbb{E}\left[\Lambda(t, x) J_{i j}^{2}(t, x)\right]$, $\phi_{i j r}(x, t)=\mathbb{E}\left[\Lambda(t, x) J_{i j r}^{3}(t, x)\right], x \in \mathbb{R}^{n}, t \in(0,1)$, are continuous and bounded on $\mathbb{R}^{n} \times(0,1)$.

Proof. Let us deal with $\phi_{i}$. We define the deterministic function $K$ : $\mathbb{R}^{n} \times \mathbb{R}^{n} \times[0,1] \rightarrow \mathbb{R}$ by

$K(x, z, t)=\left\langle D_{E_{0}} f\left(Y_{t}^{z}\right), E_{0}\left(x-Y_{t}^{z}\right)\right\rangle+\frac{1}{2}\left\langle D_{E_{0}}^{2} f\left(Y_{t}^{z}\right)\left[E_{0}\left(x-Y_{t}^{z}\right)\right], E_{0}\left(x-Y_{t}^{z}\right)\right\rangle$ and $g_{i}: \mathbb{R}^{n} \times \mathbb{R}^{n} \times(0,1] \rightarrow \mathbb{R}$ by

$$
g_{i}(x, z, t)=\mathbb{E}\left[K\left(X_{t}^{x}, z, t\right) J_{i}^{1}(t, x)\right], \quad x, z \in \mathbb{R}^{n}, t \in(0,1] .
$$

Note that $\phi_{i}(x, t)=g_{i}(x, x, t)$ for $x \in \mathbb{R}^{n}$ and $t \in(0,1)$. We first prove that

$$
g_{i}(x, z, t)=D_{i}\left(\mathbb{E}\left[K\left(X_{t}^{(\cdot)}, z, t\right)\right]\right)(x)=\mathbb{E}\left[\left\langle D_{x} K\left(X_{t}^{x}, z, t\right), \eta_{i}(t, x)\right\rangle\right]
$$

for $x, z \in \mathbb{R}^{n}$ and $t \in(0,1)$ (here $D_{x_{i}}=D_{i}$ denotes the partial derivative with respect to $e_{i}$ and $D_{x}$ denotes the gradient in the $x$-variable; $\eta_{i}$ is introduced in Lemma 3.6). To this end, observe that

$$
\begin{array}{r}
|K(x, z, t)|+\left|D_{x_{i}} K(x, z, t)\right|+\left|D_{x_{i} x_{j}}^{2} K(x, z, t)\right|+\left|D_{x_{i} x_{j} x_{r}}^{3} K(x, z, t)\right| \\
\leq 8\|f\|_{\gamma, d}\left(1+\left|E_{0}\left(x-Y_{t}^{z}\right)\right|^{2}\right)
\end{array}
$$


for $t \in[0,1], x, z \in \mathbb{R}^{n}$, and $i, j, r \in\{1, \ldots, n\}$. Furthermore, an application of the Gronwall lemma shows that

$$
\left|X_{t}^{x}\right| \leq e^{\left(\|A\|_{L}+\|D F\|_{0}\right)}\left(|x|+|F(0)|+\|\sqrt{Q}\|_{L} \sup _{s \leq 1}\left|W_{s}\right|\right)
$$

for $t \in[0,1]$ and $x \in \mathbb{R}^{n}, \mathbb{P}$-a.s. By (3.21) and (3.22), using Lemma 3.6, we get the existence of the partial derivatives

$$
D_{x_{i}}\left(\mathbb{E}\left[K\left(X_{t}^{(\cdot)}, z, t\right)\right]\right)(x)=\mathbb{E}\left[\left\langle D_{x} K\left(X_{t}^{x}, z, t\right), \eta_{i}(t, x)\right\rangle\right]
$$

for $x, z \in \mathbb{R}^{n}, t \in(0,1)$, and $1 \leq i \leq n$. To obtain (3.20), we consider test functions $\varphi_{m} \in C_{0}^{\infty}\left(\mathbb{R}^{n}\right)$ such that $0 \leq \varphi_{m} \leq 1$ for $m \in \mathbb{N}, \varphi_{m}(x)=1$ when $|x| \leq n, \varphi_{m}(x)=0$ when $|x|>m+1$, and $\left|D \varphi_{m}(x)\right| \leq 1$ for $x \in \mathbb{R}^{n}$ and $m \in \mathbb{N}$. By Theorem 3.1 and Lemma 3.6, we know that, for $x, z \in \mathbb{R}^{n}, t \in$ $(0,1)$ and $m \in \mathbb{N}$,

$$
\begin{aligned}
& D_{x_{i}}\left(\mathbb{E}\left[K\left(X_{t}^{(\cdot)}, z, t\right) \varphi_{m}\left(X_{t}^{(\cdot)}\right)\right]\right)(x)=\mathbb{E}\left[K\left(X_{t}^{x}, z, t\right) \varphi_{m}\left(X_{t}^{x}\right) J_{i}^{1}(t, x)\right] \\
& \quad=\mathbb{E}\left[\left\langle D_{x} K\left(X_{t}^{x}, z, t\right), \eta_{i}(t, x)\right\rangle \varphi_{m}\left(X_{t}^{x}\right)+K\left(X_{t}^{x}, z, t\right)\left\langle D \varphi_{m}\left(X_{t}^{x}\right), \eta_{i}(t, x)\right\rangle\right] .
\end{aligned}
$$

Passing to the limit as $m \rightarrow \infty$, we get (3.20) by the dominated convergence theorem. By (3.20), (3.21) and Lemma 3.6, we have (setting $z=x$ )

$$
\begin{aligned}
\left|\phi_{i}(x, t)\right| & =\left|g_{i}(x, x, t)\right| \leq \mathbb{E}\left[\left|D_{x_{i}} K\left(X_{t}^{x}, x, t\right)\right|\left|\eta_{i}(t, x)\right|\right] \\
& \leq 8\left\|\eta_{i}\right\|_{L^{\infty}}\|f\|_{\gamma, d}\left(1+\mathbb{E}\left[\left|E_{0}\left(X_{t}^{x}-Y_{t}^{x}\right)\right|^{2}\right]\right)
\end{aligned}
$$

for any $x \in \mathbb{R}^{n}$ and $t \in(0,1)$. Applying Lemma 3.5, we get

$$
\sup _{x \in \mathbb{R}^{n}, t \in(0,1)}\left|\phi_{i}(x, t)\right| \leq 8 c\left\|\eta_{i}\right\|_{L^{\infty}}\|f\|_{\gamma, d} \sup _{t \in(0,1)}|1+t| \leq 16 c\left\|\eta_{i}\right\|_{L^{\infty}}\|f\|_{\gamma, d} .
$$

To deal with $\phi_{i j}$ and $\phi_{i j r}$ we proceed similarly. For $\phi_{i j}$ we introduce

$$
\begin{aligned}
& g_{i j}(x, z, t)=\mathbb{E}\left[K\left(X_{t}^{x}, z, t\right) J_{i j}^{2}(t, x)\right]=D_{i j}^{2}\left(\mathbb{E}\left[K\left(X_{t}^{(\cdot)}, z, t\right)\right]\right)(x) \\
& \quad=\mathbb{E}\left[\left\langle D_{x}^{2} K\left(X_{t}^{x}, z, t\right)\left[\eta_{j}(t, x)\right], \eta_{i}(t, x)\right\rangle+\left\langle D_{x} K\left(X_{t}^{x}, z, t\right), \eta_{i j}(t, x)\right\rangle\right] .
\end{aligned}
$$

Since $\phi_{i j}(x, t)=g_{i j}(x, x, t)$, we obtain the assertion for $\phi_{i j}$ using (3.21) and Lemmas 3.6 and 3.5, as before. To deal with $\phi_{i j r}$ we introduce $g_{i j r}(x, z, t)=$ $\mathbb{E}\left[K\left(X_{t}^{x}, z, t\right) J_{i j r}^{3}(t, x)\right]$. Note that

$$
\begin{aligned}
& g_{i j r}(x, z, t)=\mathbb{E}\left[\left\langle D_{x}^{3} K\left(X_{t}^{x}, z, t\right)\left[\eta_{r}(t, x)\right]\left[\eta_{j}(t, x)\right], \eta_{i}(t, x)\right\rangle\right. \\
& \quad+\left\langle D_{x}^{2} K\left(X_{t}^{x}, z, t\right)\left[\eta_{j r}(t, x)\right], \eta_{i}(t, x)\right\rangle+\left\langle D_{x}^{2} K\left(X_{t}^{x}, z, t\right)\left[\eta_{j}(t, x)\right], \eta_{i r}(t, x)\right\rangle \\
& \left.\quad+\left\langle D_{x}^{2} K\left(X_{t}^{x}, z, t\right)\left[\eta_{r}(t, x)\right], \eta_{i j}(t, x)\right\rangle+\left\langle D_{x} K\left(X_{t}^{x}, z, t\right), \eta_{i j r}(t, x)\right\rangle\right] .
\end{aligned}
$$

Since $\phi_{i j r}(x, t)=g_{i j r}(x, x, t)$, we get the assertion for $\phi_{i j r}$ proceeding as for $\phi_{i}$ and $\phi_{i j}$. The proof is complete.

Proof of Theorem 3.3. Thanks to Corollary 3.2, it is enough to prove all the estimates for $0<t<1$. Indeed, concerning (iv) in (3.10), we have, for 
$t \geq 1$ and $f \in \mathcal{C}_{d}^{\gamma}\left(\mathbb{R}^{n}\right)$

$$
\begin{aligned}
\left\|P_{t} f\right\|_{\gamma, d} & =\sum_{m=0}^{k} \sup _{z \in \mathbb{R}^{n}}\left\|\left(P_{t} f\right)(z+\cdot)\right\|_{C_{b}^{\gamma /(2 m+1)}\left(E_{m}\left(\mathbb{R}^{n}\right)\right)} \\
& \leq c^{\prime} \sum_{m=0}^{k}\left(\|f\|_{0}+\sup _{z \in \mathbb{R}^{n}}\left\|\left(P_{t} f\right)(z+\cdot)\right\|_{C_{b}^{3}\left(E_{m}\left(\mathbb{R}^{n}\right)\right)}\right) \leq c_{2}\|f\|_{0} .
\end{aligned}
$$

We will show the estimates for $\gamma \in(2,3)$. Indeed, the cases of $\gamma \in(0,1)$ and $\gamma \in(1,2)$ can be handled similarly and are even simpler. Alternatively, once we have proved the estimates for $\gamma \in(2,3)$, the remaining estimates can be obtained by an interpolation argument. Let us briefly explain this method, which has also been used in the proof of [21, Theorem 3.4]. We assume that (i)-(iv) hold for $\gamma=5 / 2$ and show that they also hold for any fixed $\gamma^{\prime} \in(0,2), \gamma^{\prime} \neq 1$. By [21, Theorem 2.2], we know in particular that

$$
\left(C_{b}\left(\mathbb{R}^{n}\right), \mathcal{C}_{d}^{5 / 2}\left(\mathbb{R}^{n}\right)\right)_{2 \gamma^{\prime} / 5, \infty}=\mathcal{C}_{d}^{\gamma^{\prime}}\left(\mathbb{R}^{n}\right) .
$$

To be precise, (3.23) is proved in [21] with $C_{b}\left(\mathbb{R}^{n}\right)$ denoting the Banach space of all real continuous bounded functions on $\mathbb{R}^{n}$. However, the same proof works when we consider $C_{b}\left(\mathbb{R}^{n}\right)$ as the space of all real uniformly continuous and bounded functions. Concerning estimate (iv) in (3.10), by (3.23) and [23, Proposition 1.2.6] we get

$$
\begin{aligned}
& \left\|P_{t}\right\|_{L\left(\mathcal{C}_{d}^{\gamma^{\prime}}\left(\mathbb{R}^{n}\right), \mathcal{C}_{d}^{\gamma^{\prime}}\left(\mathbb{R}^{n}\right)\right)} \\
& \quad \leq\left(\left\|P_{t}\right\|_{L\left(C_{b}\left(\mathbb{R}^{n}\right), C_{b}\left(\mathbb{R}^{n}\right)\right)}\right)^{1-2 \gamma^{\prime} / 5}\left(\left\|P_{t}\right\|_{L\left(\mathcal{C}_{d}^{5 / 2}\left(\mathbb{R}^{n}\right), \mathcal{C}_{d}^{5 / 2}\left(\mathbb{R}^{n}\right)\right)}\right)^{2 \gamma^{\prime} / 5} \leq C
\end{aligned}
$$

for $t \geq 0$. As for (iii), we fix $x \in \mathbb{R}^{n}$ and $t \in(0,1]$, and define the linear operator $T_{x, t}: \mathcal{C}_{d}^{\gamma^{\prime}}\left(\mathbb{R}^{n}\right) \rightarrow \mathbb{R}$ by $T_{x, t} f:=D_{i j r}^{3} P_{t} f(x)$ for any $f \in \mathcal{C}_{d}^{\gamma^{\prime}}\left(\mathbb{R}^{n}\right)$. We have

$$
\begin{aligned}
\left\|T_{x, t}\right\|_{L\left(\mathcal{C}_{d}^{\gamma^{\prime}}\left(\mathbb{R}^{n}\right), \mathbb{R}\right)} & \leq\left(\left\|T_{x, t}\right\|_{L\left(C_{b}\left(\mathbb{R}^{n}\right), \mathbb{R}\right)}\right)^{1-2 \gamma^{\prime} / 5}\left(\left\|T_{x, t}\right\|_{L\left(C_{d}^{5 / 2}\left(\mathbb{R}^{n}\right), \mathbb{R}\right)}\right)^{2 \gamma^{\prime} / 5} \\
& \leq c t^{-\left(3 / 2+h+h^{\prime}+h^{\prime \prime}\right)+\gamma^{\prime} / 2}
\end{aligned}
$$

for $t \in(0,1]$ (uniformly in $x \in \mathbb{R}^{n}$ ). In a similar way, one can prove (i) and (ii) for $\gamma^{\prime}$.

Proof of (3.10)(i) for $t \in(0,1), \gamma \in(2,3)$ and $i \in I_{h}$. We start from (3.6) and write $D_{i} P_{t} f(x)=\Lambda_{1}(t, x)+\Lambda_{2}(t, x)$ where

$$
\Lambda_{1}(t, x)=\mathbb{E}\left[\left\{f\left(X_{t}^{x}\right)-f\left(E_{0} X_{t}^{x}+\sum_{h=1}^{k} E_{h} Y_{t}^{x}\right)\right\} J_{i}^{1}(t, x)\right],
$$




$$
\Lambda_{2}(t, x)=\mathbb{E}\left[f\left(E_{0} X_{t}^{x}+\sum_{h=1}^{k} E_{h} Y_{t}^{x}\right) J_{i}^{1}(t, x)\right],
$$

where $\left(Y_{t}^{x}\right)$ is defined in (3.12). Let us deal with $\Lambda_{1}$ and $\Lambda_{2}$ separately. Using (3.7), (3.8) and Lemma 3.5, we have (since $0<\gamma /(2 m+1)<1$ if $m=1, \ldots, k)$

$$
\begin{aligned}
\left|\Lambda_{1}(t, x)\right| & \leq c\|f\|_{\gamma, d} \mathbb{E}\left[\left\{\sum_{m=1}^{k}\left|E_{m}\left(X_{t}^{x}-Y_{t}^{x}\right)\right|^{\gamma /(2 m+1)}\right\}\left|J_{i}^{1}(t, x)\right|\right] \\
& \leq c^{\prime}\|f\|_{\gamma, d}\left(\mathbb{E}\left\|X_{t}^{x}-Y_{t}^{x}\right\|^{2 \gamma}\right)^{1 / 2}\left(\mathbb{E}\left|J_{i}^{1}(t, x)\right|^{2}\right)^{1 / 2} \\
& \leq c_{2}\|f\|_{\gamma, d} t^{\gamma / 2} t^{-(h+1 / 2)}=c_{2}\|f\|_{\gamma, d} t^{(\gamma-1) / 2-h}
\end{aligned}
$$

for $t \in(0,1)$, uniformly in $x \in \mathbb{R}^{n}$. Let us turn to the more difficult term $\Lambda_{2}$. We write $\Lambda_{2}(t, x)=\Lambda_{21}(t, x)+\Lambda_{22}(t, x)$, where

$$
\begin{aligned}
& \Lambda_{21}(t, x)=\mathbb{E} {\left[\left(f\left(E_{0} X_{t}^{x}+\sum_{m=1}^{k} E_{m} Y_{t}^{x}\right)-f\left(Y_{t}^{x}\right)\right.\right.} \\
&-\left\langle D_{E_{0}} f\left(Y_{t}^{x}\right), E_{0}\left(X_{t}^{x}-Y_{t}^{x}\right)\right\rangle \\
&\left.\left.-\frac{1}{2}\left\langle D_{E_{0}}^{2} f\left(Y_{t}^{x}\right)\left[E_{0}\left(X_{t}^{x}-Y_{t}^{x}\right)\right], E_{0}\left(X_{t}^{x}-Y_{t}^{x}\right)\right\rangle\right) J_{i}^{1}(t, x)\right], \\
& \Lambda_{22}(t, x)=\mathbb{E}\left[\left(\left\langle D_{E_{0}} f\left(Y_{t}^{x}\right), E_{0}\left(X_{t}^{x}-Y_{t}^{x}\right)\right\rangle\right.\right. \\
&\left.\left.+\frac{1}{2}\left\langle D_{E_{0}}^{2} f\left(Y_{t}^{x}\right)\left[E_{0}\left(X_{t}^{x}-Y_{t}^{x}\right)\right], E_{0}\left(X_{t}^{x}-Y_{t}^{x}\right)\right\rangle\right) J_{i}^{1}(t, x)\right]
\end{aligned}
$$

(see (2.6)). Note that, since $\left(Y_{t}^{x}\right)$ is deterministic,

$$
\mathbb{E}\left[f\left(Y_{t}^{x}\right) J_{i}^{1}(t, x)\right]=f\left(Y_{t}^{x}\right) \mathbb{E}\left[J_{i}^{1}(t, x)\right]=f\left(Y_{t}^{x}\right) D_{i}\left(P_{t} 1\right)(x)=0
$$

for $x \in \mathbb{R}^{n}$ and $t>0$. To estimate $\Lambda_{21}$, note that $f(x+\cdot) \in C_{b}^{\gamma}\left(E_{0}\left(\mathbb{R}^{n}\right)\right)$, $\gamma \in(2,3)$, uniformly in $x$. By the mean value theorem, we have

$$
\begin{aligned}
\sup _{x \in \mathbb{R}^{n}}\left|\Lambda_{21}(t, x)\right| & \leq\|f\|_{\gamma, d} \sup _{x \in \mathbb{R}^{n}} \mathbb{E}\left[\left|E_{0}\left(X_{t}^{x}-Y_{t}^{x}\right)\right|^{\gamma}\left|J_{i}^{1}(t, x)\right|\right] \\
& \leq\|f\|_{\gamma, d} \sup _{x \in \mathbb{R}^{n}}\left(\mathbb{E}\left[\left|E_{0}\left(X_{t}^{x}-Y_{t}^{x}\right)\right|^{2 \gamma}\right]\right)^{1 / 2} \cdot \sup _{x \in \mathbb{R}^{n}}\left(\mathbb{E}\left|J_{i}^{1}(t, x)\right|^{2}\right)^{1 / 2} \\
& \leq c_{3}\|f\|_{\gamma, d} t^{(\gamma-1) / 2-h}
\end{aligned}
$$

(see also (3.14)). Finally, using Lemma 3.7, we infer

$$
\sup _{x \in \mathbb{R}^{n}, t \in(0,1)}\left|\Lambda_{22}(t, x)\right|=c_{4}<\infty .
$$

Proof of (3.10)(ii) and (iii) for $t \in(0,1)$ and $\gamma \in(2,3)$. These estimates can be obtained proceeding as in the proof of (3.10)(i). We only prove (ii). Let $i \in I_{h}$ and $j \in I_{h^{\prime}}$. We write

$$
D_{i j}^{2} P_{t} f(x)=\tilde{\Lambda}_{1}(t, x)+\tilde{\Lambda}_{21}(t, x)+\tilde{\Lambda}_{22}(t, x),
$$


where

$$
\begin{aligned}
& \tilde{\Lambda}_{1}(t, x)=\mathbb{E}\left[\left\{f\left(X_{t}^{x}\right)-f\left(E_{0} X_{t}^{x}+\sum_{h=1}^{k} E_{h} Y_{t}^{x}\right)\right\} J_{i j}^{2}(t, x)\right], \\
& \tilde{\Lambda}_{21}(t, x)=\mathbb{E}\left[\left(f\left(E_{0} X_{t}^{x}+\sum_{h=1}^{k} E_{h} Y_{t}^{x}\right)-f\left(Y_{t}^{x}\right)\right.\right. \\
&-\left\langle D_{E_{0}} f\left(Y_{t}^{x}\right), E_{0}\left(X_{t}^{x}-Y_{t}^{x}\right)\right\rangle \\
&\left.\left.-\frac{1}{2}\left\langle D_{E_{0}}^{2} f\left(Y_{t}^{x}\right)\left[E_{0}\left(X_{t}^{x}-Y_{t}^{x}\right)\right], E_{0}\left(X_{t}^{x}-Y_{t}^{x}\right)\right\rangle\right) J_{i j}^{2}(t, x)\right], \\
& \tilde{\Lambda}_{22}(t, x)=\mathbb{E}\left[\left(\left\langle D_{E_{0}} f\left(Y_{t}^{x}\right), E_{0}\left(X_{t}^{x}-Y_{t}^{x}\right)\right\rangle\right.\right. \\
&\left.\left.+\frac{1}{2}\left\langle D_{E_{0}}^{2} f\left(Y_{t}^{x}\right)\left[E_{0}\left(X_{t}^{x}-Y_{t}^{x}\right)\right], E_{0}\left(X_{t}^{x}-Y_{t}^{x}\right)\right\rangle\right) J_{i j}^{2}(t, x)\right],
\end{aligned}
$$

for $t \in(0,1)$, and $x \in \mathbb{R}^{n}$. We have (using (3.7), (3.8), and Lemmas 3.5 and 3.7)

$$
\begin{aligned}
\sup _{x \in \mathbb{R}^{n}}\left|\tilde{\Lambda}_{1}(t, x)\right| & \leq c\|f\|_{\gamma, d} \sup _{x \in \mathbb{R}^{n}} \mathbb{E}\left[\left\{\sum_{m=1}^{k}\left|E_{m}\left(X_{t}^{x}-Y_{t}^{x}\right)\right|^{\gamma /(2 m+1)}\right\}\left|J_{i j}^{2}(t, x)\right|\right] \\
& \leq c_{2}\|f\|_{\gamma, d} t^{(\gamma-2) / 2-h-h^{\prime}} .
\end{aligned}
$$

By the mean value theorem, we find

$$
\begin{aligned}
\sup _{x \in \mathbb{R}^{n}}\left|\tilde{\Lambda}_{21}(t, x)\right| & \leq\|f\|_{\gamma, d} \sup _{x \in \mathbb{R}^{n}} \mathbb{E}\left[\left|E_{0}\left(X_{t}^{x}-Y_{t}^{x}\right)\right|^{\gamma}\left|J_{i j}^{2}(t, x)\right|\right] \\
& \leq c\|f\|_{\gamma, d} t^{(2 \gamma-2) / 2-h-h^{\prime}} .
\end{aligned}
$$

Using Lemma 3.7, we infer

$$
\sup _{x \in \mathbb{R}^{n}, t \in(0,1)}\left|\tilde{\Lambda}_{22}(t, x)\right|=c_{5}<\infty .
$$

Proof of $(3.10)$ (iv) for $t \in(0,1)$ and $\gamma \in(2,3)$. We have to show that, for any $0 \leq h \leq k, f \in \mathcal{C}_{d}^{\gamma}\left(\mathbb{R}^{n}\right), t \in(0,1)$,

$$
\sup _{x \in \mathbb{R}^{n}}\left\|P_{t} f(x+\cdot)\right\|_{C_{b}^{\gamma / 2 h+1}\left(E_{h}\left(\mathbb{R}^{n}\right)\right)} \leq c\|f\|_{\gamma, d} .
$$

Fix $h$ and $f \in \mathcal{C}_{d}^{\gamma}\left(\mathbb{R}^{n}\right)$ and consider

$$
\triangle_{v_{h}}^{3}\left(P_{t} f\right)(x)=P_{t} f(x)-3 P_{t} f\left(x+v_{h}\right)+3 P_{t} f\left(x+2 v_{h}\right)-P_{t} f\left(x+3 v_{h}\right)
$$

for $x \in \mathbb{R}^{n}$ and $v_{h} \in E_{h}\left(\mathbb{R}^{n}\right)$ with $\left|v_{h}\right| \leq 1$ and $v_{h} \neq 0$. By (2.8) the assertion (3.24) is equivalent to the estimate

$$
\sup _{x \in \mathbb{R}^{n}}\left|\triangle_{v_{h}}^{3}\left(P_{t} f\right)(x)\right| \leq c_{1}\|f\|_{\gamma, d}\left|v_{h}\right|^{\gamma /(2 h+1)},
$$

where $c_{1}$ is independent of $f, t$ and $v_{h}$. We prove (3.25) considering first the case of $\left|v_{h}\right| \leq t^{(2 h+1) / 2}<1$ and then $1 \geq\left|v_{h}\right|>t^{(2 h+1) / 2}$ (cf. [21, p. 148]). 
(a) Let $\left|v_{h}\right| \leq t^{(2 h+1) / 2}<1$. Using the mean value theorem and (3.10)(iii), we get, for any $t \in(0,1)$,

$$
\begin{aligned}
\sup _{x \in \mathbb{R}^{n}}\left|\triangle_{v_{h}}^{3}\left(P_{t} f\right)(x)\right| & \leq \sup _{x \in \mathbb{R}^{n}, i, j, r \in I_{h}}\left\|D_{i j r}^{3} P_{t} f\right\|_{0}\left|v_{h}\right|^{3} \\
& \leq c \frac{1}{t^{3 h+(3-\gamma) / 2}}\|f\|_{\gamma, d}\left|v_{h}\right|^{3} \\
& \leq c \frac{1}{\left|v_{h}\right|^{\frac{2}{2 h+1}} \frac{6 h+3-\gamma}{2}}\|f\|_{\gamma, d}\left|v_{h}\right|^{3}=c\|f\|_{\gamma, d}\left|v_{h}\right|^{\gamma /(2 h+1)} .
\end{aligned}
$$

(b) Let $1 \geq\left|v_{h}\right|>t^{(2 h+1) / 2}$. We first estimate $\left\|e^{t A} v_{h}\right\|$. We use the estimates

$$
\left\|E_{i} e^{t A} E_{h}\right\|_{L} \leq \begin{cases}c t^{i-h}, & 0 \leq h \leq i \leq k, \\ c t, & 0 \leq i<h \leq k,\end{cases}
$$

for $t \in[0,1]$ (see [21, Lemma 3.1]) where $c=c(A)>0$. Since $t \leq\left|v_{h}\right|^{2 /(2 h+1)}$ $\leq 1$, we get

$$
\begin{aligned}
\left\|e^{t A} v_{h}\right\| & =\sum_{i=0}^{h-1}\left|E_{i} e^{t A} E_{h} v_{h}\right|^{\frac{1}{2 i+1}}+\sum_{i=h}^{k}\left|E_{i} e^{t A} E_{h} v_{h}\right|^{\frac{1}{2 i+1}} \\
& \leq c_{1} \sum_{i=0}^{h-1}\left|t v_{h}\right|^{\frac{1}{2 i+1}}+c_{1} \sum_{i=h}^{k} t^{\frac{i-h}{2 i+1}}\left|v_{h}\right|^{\frac{1}{2 i+1}} \\
& \leq c_{1} h\left|v_{h}\right|^{\frac{1}{2 h+1}}+c_{1} \sum_{i=h}^{k}\left|v_{h}\right|^{\frac{1}{2 i+1}+\frac{i-h}{2 i+1} \frac{2}{2 h+1}} \\
& \leq c_{1}(k+1)\left|v_{h}\right|^{\frac{1}{2 h+1}}
\end{aligned}
$$

To finish the proof we will use the Girsanov theorem (see (3.4)). First note that

$$
\begin{aligned}
\triangle_{v_{h}}^{3}\left(P_{t} f\right)(x)= & \mathbb{E}\left[f\left(Z_{t}^{x}\right) \Phi(t, x)-3 f\left(Z_{t}^{x+v_{h}}\right) \Phi\left(t, x+v_{h}\right)\right. \\
& \left.+3 f\left(Z_{t}^{x+2 v_{h}}\right) \Phi\left(t, x+2 v_{h}\right)-f\left(Z_{t}^{x+3 v_{h}}\right) \Phi\left(t, x+3 v_{h}\right)\right] \\
= & A_{1}(t, x)+A_{2}(t, x),
\end{aligned}
$$

where

$$
\begin{aligned}
A_{1}(t, x)= & \mathbb{E}\left[\left(f\left(Z_{t}^{x}\right)-3 f\left(Z_{t}^{x+v_{h}}\right)+3 f\left(Z_{t}^{x+2 v_{h}}\right)-f\left(Z_{t}^{x+3 v_{h}}\right)\right) \Phi(t, x)\right], \\
A_{2}(t, x)= & 3 \mathbb{E}\left[f\left(Z_{t}^{x+v_{h}}\right)\left(\Phi(t, x)-\Phi\left(t, x+v_{h}\right)\right)\right] \\
& +3 \mathbb{E}\left[f\left(Z_{t}^{x+2 v_{h}}\right)\left(\Phi\left(t, x+2 v_{h}\right)-\Phi(t, x)\right)\right] \\
& +\mathbb{E}\left[f\left(Z_{t}^{x+3 v_{h}}\right)\left(\Phi(t, x)-\Phi\left(t, x+3 v_{h}\right)\right)\right] .
\end{aligned}
$$

Let us deal with $A_{1}$. We find, for any $x \in \mathbb{R}^{n}, t \in(0,1)$, thanks to Lemma 2.1, 


$$
\begin{aligned}
\left|A_{1}(t, x)\right| & \leq \mathbb{E}\left[\left|\triangle_{e^{t A} v_{h}}^{3} f\left(e^{t A} x+Z_{t}^{0}\right)\right| \Phi(t, x)\right] \leq\|f\|_{\gamma, d}\left\|e^{t A} v_{h}\right\|^{\gamma} \\
& \leq c\|f\|_{\gamma, d}\left|v_{h}\right|^{\gamma /(2 h+1)}
\end{aligned}
$$

(in the last inequality we have used (3.27)). It remains to consider $A_{2}$. We have $A_{2}(t, x)=A_{21}(t, x)+A_{22}(t, x)$, where

$$
\begin{aligned}
& A_{21}(t, x)=\mathbb{E}\left[f ( Z _ { t } ^ { x } ) \left(\Phi(t, x)-3 \Phi\left(t, x+v_{h}\right)\right.\right. \\
&+ \\
& A_{22}(t, x)=3 \mathbb{E}\left[\left(f\left(Z_{t}^{x+v_{h}}\right)-f\left(Z_{t}^{x}\right)\right)\left(\Phi(t, x)-\Phi\left(t, x+v_{h}\right)\right)\right] \\
&+ 3 \mathbb{E}\left[\left(f\left(Z_{t}^{x+2 v_{h}}\right)-f\left(Z_{t}^{x}\right)\right)\left(\Phi\left(t, x+2 v_{h}\right)-\Phi(t, x)\right)\right] \\
&+\mathbb{E}\left[\left(f\left(Z_{t}^{x+3 v_{h}}\right)-f\left(Z_{t}^{x}\right)\right)\left(\Phi(t, x)-\Phi\left(t, x+3 v_{h}\right)\right)\right] .
\end{aligned}
$$

In order to deal with $A_{21}$, note that the map $x \mapsto \Phi(t, x)$ is three times Fréchet differentiable from $\mathbb{R}^{n}$ to $L^{1}(\Omega)$. We need to estimate the norm of the first, second and third Fréchet derivatives of $\Phi(t, \cdot)$ (the derivatives will be denoted $D_{x} \Phi(t, x), D_{x x}^{2} \Phi(t, x)$ and $D_{x x x}^{3} \Phi(t, x)$ respectively).

For any $x, h \in \mathbb{R}^{n}$, we find (setting $G=Q^{-1 / 2} F$ )

$$
\begin{aligned}
D_{x} \Phi(t, x)[h] & =\Phi(t, x) \int_{0}^{t}\left\langle D G\left(Z_{s}^{x}\right) e^{s A} h, d L_{s}^{x}\right\rangle \\
& =\Phi(t, x)\left(\int_{0}^{t}\left\langle D G\left(Z_{s}^{x}\right) e^{s A} h, d W_{s}\right\rangle-\int_{0}^{t}\left\langle D G\left(Z_{s}^{x}\right) e^{s A} h, G\left(Z_{s}^{x}\right)\right\rangle d s\right),
\end{aligned}
$$

since $L_{s}^{x}:=W_{s}-\int_{0}^{s} G\left(Z_{r}^{x}\right) d r$ for $s \in[0, t]$ (see (3.4)). By the Girsanov theorem, we have

$$
\begin{aligned}
\mathbb{E}\left|D_{x} \Phi(t, x)[h]\right| & =\mathbb{E}\left|\int_{0}^{t}\left\langle D G\left(X_{s}^{x}\right) e^{s A} h, d W_{s}\right\rangle\right| \leq e^{\|A\|_{L}}|h| t^{1 / 2}\|D G\|_{0} \\
& \leq e^{\|A\|_{L}}|h|\|D G\|_{0}, \quad t \in[0,1], h \in \mathbb{R}^{n} .
\end{aligned}
$$

It follows that $\left\|D_{x} \Phi(t, x)\right\|_{L\left(\mathbb{R}^{n}, L^{1}(\Omega)\right)} \leq e^{\|A\|}\left\|Q_{0}^{-1 / 2}\right\|_{L(\mathbb{R} \tilde{p})}\|D F\|_{0}$ for $t \in$ $[0,1]$. Similarly, for the second Fréchet derivative we have

$$
\begin{aligned}
D_{x x}^{2} \Phi(t, x)[h][k]= & \Phi(t, x)\left(\int_{0}^{t}\left\langle D G\left(Z_{s}^{x}\right) e^{s A} h, d L_{s}^{x}\right\rangle\right)\left(\int_{0}^{t}\left\langle D G\left(Z_{s}^{x}\right) e^{s A} k, d L_{s}^{x}\right\rangle\right) \\
& +\Phi(t, x)\left(\int_{0}^{t}\left\langle D^{2} G\left(Z_{s}^{x}\right)\left[e^{s A} k\right]\left[e^{s A} h\right], d L_{s}^{x}\right\rangle\right. \\
& \left.-\int_{0}^{t}\left\langle D G\left(Z_{s}^{x}\right)\left[e^{s A} h\right], D G\left(Z_{s}^{x}\right)\left[e^{s A} k\right]\right\rangle d s\right)
\end{aligned}
$$


for $h, k \in \mathbb{R}^{n}$. It follows, by the Girsanov theorem, that

$\mathbb{E}\left|D_{x x}^{2} \Phi(t, x)[h][k]\right| \leq c_{2}|h||k|\left(\|D G\|_{0}^{2}+\left\|D^{2} G\right\|_{0}\right), \quad t \in[0,1], h, k \in \mathbb{R}^{n}$.

In a similar way, we get

$\mathbb{E}\left|D_{x x x}^{3} \Phi(t, x)[h][k][u]\right| \leq c|h||k||u|\left(\|D G\|_{0}^{3}+\|D G\|_{0}^{2}+\left\|D^{2} G\right\|_{0}^{2}+\left\|D^{3} G\right\|_{0}\right)$ $\leq C_{1}|h||k||u|, \quad t \in[0,1], h, k, u \in \mathbb{R}^{n}$,

where $C_{1}=C_{1}\left(\|A\|_{L}, \nu_{1}, \tilde{p},\|D F\|_{0},\left\|D^{2} F\right\|_{0},\left\|D^{3} F\right\|_{0}\right)>0$. Using the last estimate, we find

$$
\begin{aligned}
& \left|A_{21}(t, x)\right| \leq\|f\|_{0}\left\|\Phi(t, x)-3 \Phi\left(t, x+v_{h}\right)+3 \Phi\left(t, x+2 v_{h}\right)-\Phi\left(t, x+3 v_{h}\right)\right\|_{L^{1}(\Omega)} \\
& \quad \leq\|f\|_{0} \sup _{|u|,|h|,|k| \leq 1, x \in \mathbb{R}^{n}}\left\|D_{x x x}^{3} \Phi(t, x)[h][k][u]\right\|_{L^{1}(\Omega)}\left|v_{h}\right|^{3} \leq C_{1}\|f\|_{0}\left|v_{h}\right|^{3}
\end{aligned}
$$

for $x \in \mathbb{R}^{n}$ and $t \in[0,1]$. It remains to consider $A_{22}$. This is the sum of three terms which can be treated in the same way. Let us estimate the first term (without the factor 3). By (3.27), we find (recall that $\gamma \in(2,3)$ )

$$
\begin{aligned}
\mathbb{E}\left|\left(f\left(Z_{t}^{x+v_{h}}\right)-f\left(Z_{t}^{x}\right)\right)\left(\Phi(t, x)-\Phi\left(t, x+v_{h}\right)\right)\right| \\
\leq\|f\|_{\gamma, d}\left\|e^{t A} v_{h}\right\| \mathbb{E}\left|\Phi(t, x)-\Phi\left(t, x+v_{h}\right)\right| \\
\leq c\|f\|_{\gamma, d}\left|v_{h}\right|^{1 /(2 h+1)}\left\|\Phi(t, x)-\Phi\left(t, x+v_{h}\right)\right\|_{L^{1}(\Omega)} .
\end{aligned}
$$

By (3.28), since $\left|v_{h}\right|>t^{(2 h+1) / 2}$,

$$
\begin{aligned}
& \mathbb{E}\left|\left(f\left(Z_{t}^{x+v_{h}}\right)-f\left(Z_{t}^{x}\right)\right)\left(\Phi(t, x)-\Phi\left(t, x+v_{h}\right)\right)\right| \\
& \quad \leq e^{\|A\|_{L}}\|f\|_{\gamma, d}\left|v_{h}\right|^{1 /(2 h+1)}\left|v_{h}\right| t^{1 / 2}\|D G\|_{0} \leq c^{\prime}\left|v_{h}\right|^{2 /(2 h+1)+1}\|f\|_{\gamma, d} .
\end{aligned}
$$

We obtain $\sup _{x \in \mathbb{R}^{n}}\left|A_{22}(t, x)\right| \leq c_{3}\left|v_{h}\right|^{(3+2 h) /(2 h+1)}\|f\|_{\gamma, d}$ for $t \in(0,1)$. The estimates obtained for $A_{1}(t, x)$ and $A_{2}(t, x)$ yield (3.25).

4. Elliptic and parabolic Schauder estimates. Here we prove elliptic and parabolic Schauder estimates for $\mathcal{A}$ using the $L^{\infty}$-estimates of the previous section. Our method is different from [21, 5, 20, 31] (see Theorems 4.2 and 4.3). Before proving Schauder estimates, we show existence and uniqueness of distributional solutions for (1.2) and (1.3).

Let $\lambda>0$ and $f \in C_{b}\left(\mathbb{R}^{n}\right)$ (i.e., $f$ is uniformly continuous and bounded on $\left.\mathbb{R}^{n}\right)$. We say that a function $u \in C_{b}\left(\mathbb{R}^{n}\right)$ is a distributional solution to the elliptic equation

$$
\lambda u(x)-\mathcal{A} u(x)=f(x), \quad x \in \mathbb{R}^{n},
$$

if $\lambda \int_{\mathbb{R}^{n}} u(x) \phi(x) d x=\int_{\mathbb{R}^{n}} u(x) \mathcal{A}^{*} \phi(x) d x+\int_{\mathbb{R}^{n}} f(x) \phi(x) d x$ for any $\phi \in$ $C_{0}^{\infty}\left(\mathbb{R}^{n}\right)$, where $\mathcal{A}^{*}$ is the formal adjoint of $\mathcal{A}$, i.e.,

$\mathcal{A}^{*} \phi(x)=\frac{1}{2} \operatorname{Tr}\left(Q D^{2} \phi(x)\right)-\langle A x+F(x), D \phi(x)\rangle-\phi(x)[\operatorname{div} F(x)+\operatorname{Tr}(A)]$. 
Let $g \in C_{b}\left(\mathbb{R}^{n}\right), T>0$ and $H:[0, T] \times \mathbb{R}^{n} \rightarrow \mathbb{R}$ be a continuous bounded function. We say that a continuous bounded function $v:[0, T] \times \mathbb{R}^{n} \rightarrow \mathbb{R}$ such that $v(0, x)=g(x)$ for $x \in \mathbb{R}^{n}$ is a space-distributional solution to the parabolic Cauchy problem

$$
\left\{\begin{aligned}
\partial_{t} v(t, x) & =\mathcal{A} v(t, x)+H(t, x), \quad t \in(0, T], x \in \mathbb{R}^{n}, \\
v(0, x) & =g(x), \quad x \in \mathbb{R}^{n},
\end{aligned}\right.
$$

if the following conditions hold:

(i) $v(t, \cdot) \in C_{b}\left(\mathbb{R}^{n}\right)$ uniformly in $t \in[0, T]$ (i.e., for any $\epsilon>0$ there exists $\delta>0$ such that if $y \in \mathbb{R}^{n}$ and $|y|<\delta$, we have $\sup _{t \in[0, T], x \in \mathbb{R}^{n}} \mid v(t, x+y)-$ $v(t, x) \mid<\epsilon)$;

(ii) for any $\phi \in C_{0}^{\infty}\left(\mathbb{R}^{n}\right)$, the real function $t \mapsto \int_{\mathbb{R}^{n}} v(t, x) \phi(x) d x$ is continuously differentiable on $[0, T]$ and furthermore for $t \in[0, T]$,

$$
\frac{d}{d t}\left(\int_{\mathbb{R}^{n}} v(t, x) \phi(x) d x\right)=\int_{\mathbb{R}^{n}} v(t, x) \mathcal{A}^{*} \phi(x) d x+\int_{\mathbb{R}^{n}} H(t, x) \phi(x) d x .
$$

Theorem 4.1. Let $\lambda>0$ and $f \in C_{b}\left(\mathbb{R}^{n}\right)$. Then there exists a unique distributional solution $u \in C_{b}\left(\mathbb{R}^{n}\right)$ to the equation (4.1). Furthermore, $u$ is given by

$$
u(x)=\int_{0}^{\infty} e^{-\lambda t}\left(P_{t} f\right)(x) d t=\int_{0}^{\infty} e^{-\lambda t} P_{t} f(x) d t, \quad x \in \mathbb{R}^{n},
$$

where $P_{t}$ is the diffusion semigroup introduced in (3.2).

Let $g \in C_{b}\left(\mathbb{R}^{n}\right), T>0$ and $H:[0, T] \times \mathbb{R}^{n} \rightarrow \mathbb{R}$ be continuous and bounded. Then there exists a unique space-distributional solution $v$ to the Cauchy problem (4.2). Furthermore, setting

$$
\int_{0}^{t} P_{t-s} H(s, x) d s:=\int_{0}^{t} P_{t-s}(H(s, \cdot))(x) d s,
$$

we have

$$
v(t, x)=P_{t} g(x)+\int_{0}^{t} P_{t-s} H(s, x) d s, \quad x \in \mathbb{R}^{n}, t \in[0, T] .
$$

Proof. Uniqueness. We first consider the elliptic case. Fix $\lambda>0$ and let $u \in C_{b}\left(\mathbb{R}^{n}\right)$ be any distributional solution to (4.1) with $f=0$.

Take a $\rho \in C_{0}^{\infty}\left(\mathbb{R}^{n}\right)$ such that $\|\rho\|_{L^{1}\left(\mathbb{R}^{n}\right)}=1,0 \leq \rho \leq 1$ and $\rho(x)=0$ if $|x| \geq 1$. Define a sequence of mollifiers $\left(\rho_{m}\right) \subset C_{0}^{\infty}\left(\mathbb{R}^{n}\right)$ by $\rho_{m}(x):=$ $m^{n} \rho(m x)$ for $x \in \mathbb{R}^{n}$ and $m \in \mathbb{N}$. Consider the functions $u_{m} \in C_{b}^{\infty}\left(\mathbb{R}^{n}\right)$ obtained by convolution of $u$ with $\rho_{m}$, i.e., $u_{m}=u * \rho_{m}$. Setting $B(x):=$ 
$A x+F(x), x \in \mathbb{R}^{n}$, we use the identity

$$
\begin{array}{r}
\mathcal{A}^{*}\left[\rho_{m}(x-\cdot)\right](y)+\left\langle B(x)-B(y), D \rho_{m}(x-y)\right\rangle+\rho_{m}(x-y) \operatorname{div} B(y) \\
=\mathcal{A}\left[\rho_{m}(\cdot-y)\right](x), \quad x, y \in \mathbb{R}^{n},
\end{array}
$$

to get

$$
\begin{aligned}
\mathcal{A} u_{m}(x) & =\int_{\mathbb{R}^{n}} u(y) \mathcal{A}\left[\rho_{m}(\cdot-y)\right](x) d y \\
& =\int_{\mathbb{R}^{n}} u(y) \mathcal{A}^{*}\left[\rho_{m}(x-\cdot)\right](y) d y+R_{m, 1}(x)+R_{m, 2}(x) \\
& =\int_{\mathbb{R}^{n}} \lambda u(y) \rho_{m}(x-y) d y+R_{m, 1}(x)+R_{m, 2}(x),
\end{aligned}
$$

where

$$
\begin{aligned}
& R_{m, 1}(x)=\int_{\mathbb{R}^{n}} u(y) \operatorname{div} B(y) \rho_{m}(x-y) d y, \\
& R_{m, 2}(x)=\int_{\mathbb{R}^{n}} u(y)\left\langle B(x)-B(y), D \rho_{m}(x-y)\right\rangle d y .
\end{aligned}
$$

Changing variable as in $[20$, p. 559], we obtain

$$
R_{m, 2}(x)=m \int_{\mathbb{R}^{n}} u(x-z / m)\langle B(x)-B(x-z / m), D \rho(z)\rangle d z .
$$

It follows that $R_{m, 2}$ converges as $m \rightarrow \infty$, uniformly on $\mathbb{R}^{n}$, to the function

$$
x \mapsto u(x) \sum_{i, k=1}^{n} \int_{\mathbb{R}^{n}} D_{k} B_{i}(x) z_{k} D_{i} \rho(z) d z=-u(x) \operatorname{div} B(x) .
$$

On the other hand, it is easy to see that $R_{m, 1}$ converges as $m \rightarrow \infty$, uniformly on $\mathbb{R}^{n}$, to $u \operatorname{div} B$. It follows that $\lim _{m \rightarrow \infty}\left(R_{m, 1}+R_{m, 2}\right)=0$ in $C_{b}\left(\mathbb{R}^{n}\right)$. Hence we have obtained

$$
\lim _{m \rightarrow \infty}\left(\left\|\mathcal{A} u_{m}-\lambda u\right\|_{0}+\left\|u_{m}-u\right\|_{0}\right)=0 .
$$

By the classical maximum principle (see [3]) we deduce that $\left\|u_{m}\right\|_{0} \leq$ $(1 / \lambda)\left\|\lambda u_{m}-\mathcal{A} u_{m}\right\|_{0}$. Letting $m \rightarrow \infty$, we find that $\|u\|_{0}=0$, and this gives the assertion.

We now prove uniqueness in the parabolic case. To this end, we take $H=0$ and $g=0$ in (4.2) and consider any space-distributional solution $v$. We introduce as before a sequence of mollifiers $\left(\rho_{m}\right) \subset C_{0}^{\infty}\left(\mathbb{R}^{n}\right)$ and define

$$
v_{m}(t, x)=\int_{\mathbb{R}^{n}} v(t, y) \rho_{m}(x-y) d y, \quad t \in[0, T], x \in \mathbb{R}^{n}, m \in \mathbb{N} .
$$

It is clear that $v_{m}$ is continuous and bounded on $[0, T] \times \mathbb{R}^{n}$. Furthermore, it has continuous and bounded spatial partial derivatives of any order on 
$[0, T] \times \mathbb{R}^{n}$. Thanks to assumption (i), $v_{m}$ converges to $v$ as $m \rightarrow \infty$ uniformly on $[0, T] \times \mathbb{R}^{n}$.

By (4.3), for $t \in[0, T]$ and $x \in \mathbb{R}^{n}$, we have

$$
\begin{aligned}
\partial_{t} v_{m}(t, x) & =\int_{\mathbb{R}^{n}} v(t, y) \mathcal{A}^{*}\left[\rho_{m}(x-\cdot)\right](y) d y \\
& =\int_{\mathbb{R}^{n}} v(t, y) \mathcal{A}\left[\rho_{m}(\cdot-y)\right](x) d y+S_{m, 1}(t, x)+S_{m, 2}(t, x) \\
& =\mathcal{A} v_{m}(t, x)+S_{m, 1}(t, x)+S_{m, 2}(t, x),
\end{aligned}
$$

where

$$
\begin{aligned}
& S_{m, 1}(t, x)=-\int_{\mathbb{R}^{n}} v(t, y) \operatorname{div} B(y) \rho_{m}(x-y) d y, \\
& S_{m, 2}(t, x)=-\int_{\mathbb{R}^{n}} v(t, y)\left\langle B(x)-B(y), D \rho_{m}(x-y)\right\rangle d y .
\end{aligned}
$$

Note that $\lim _{m \rightarrow \infty} \sup _{t \in[0, T], x \in \mathbb{R}^{n}}\left|S_{m, 1}(t, x)+S_{m, 2}(t, x)\right|=0$. Furthermore, since $v_{m}$ is a classical solution to

$$
\left\{\begin{aligned}
\partial_{t} v_{m}(t, x) & =\mathcal{A} v_{m}(t, x)+S_{m, 1}(t, x)+S_{m, 2}(t, x), \quad t \in(0, T], x \in \mathbb{R}^{n}, \\
v_{m}(0, x) & =0, \quad x \in \mathbb{R}^{n},
\end{aligned}\right.
$$

by the classical parabolic maximum principle (see [15, Chapter 8$]$ ) we have

$$
\sup _{t \in[0, T], x \in \mathbb{R}^{n}}\left|v_{m}(t, x)\right| \leq T \sup _{t \in[0, T], x \in \mathbb{R}^{n}}\left|S_{m, 1}(t, x)+S_{m, 2}(t, x)\right| .
$$

Letting $m \rightarrow \infty$ we conclude that $v=0$ and this proves the assertion.

Existence. We first consider the elliptic case and prove that $u$ given in (4.4) is the distributional solution. It is clear that $u \in C_{b}\left(\mathbb{R}^{n}\right)$. In the following computations we will use the fact that the classical partial derivative $\partial_{t}\left(P_{t} f\right)(x)$ exists for $t>0$ and $x \in \mathbb{R}^{n}$, and $\partial_{t}\left(P_{t} f\right)(x)=\mathcal{A}\left(P_{t} f\right)(x)$ (see $[29$, Section 4]).

By Corollary 3.2 we deduce that, for any $M>0$, there exists $C_{M}>0$ such that

$$
\sup _{|x| \leq M}\left|\mathcal{A}\left(P_{t} f\right)(x)\right| \leq C_{M}\left(t^{-(1+k)}+1\right)\|f\|_{0}, \quad t>0, f \in C_{b}\left(\mathbb{R}^{n}\right) .
$$

For any $\phi \in C_{0}^{\infty}\left(\mathbb{R}^{n}\right)$, applying the Fubini theorem, we obtain

$$
\begin{aligned}
\int_{\mathbb{R}^{n}} u(x) \mathcal{A}^{*} \phi(x) d x & =\int_{0}^{\infty} e^{-\lambda t} d t \int_{\mathbb{R}^{n}} \mathcal{A} P_{t} f(x) \phi(x) d x \\
& =\lim _{\epsilon \rightarrow 0^{+}} \int_{\epsilon}^{\infty} e^{-\lambda t} d t \int_{\mathbb{R}^{n}} \mathcal{A} P_{t} f(x) \phi(x) d x
\end{aligned}
$$




$$
\begin{aligned}
& =\lim _{\epsilon \rightarrow 0^{+}} \int_{\epsilon}^{\infty} e^{-\lambda t} d t \int_{\mathbb{R}^{n}} \partial_{t} P_{t} f(x) \phi(x) d x \\
& =\lim _{\epsilon \rightarrow 0^{+}} \int_{\mathbb{R}^{n}} \phi(x) d x \int_{\epsilon}^{\infty} e^{-\lambda t} \partial_{t} P_{t} f(x) d t \\
& =\lim _{\epsilon \rightarrow 0^{+}} \int_{\mathbb{R}^{n}}\left(-e^{-\lambda \epsilon} P_{\epsilon} f(x)+\lambda \int_{\epsilon}^{\infty} e^{-\lambda t} P_{t} f(x) d t\right) \phi(x) \\
& =\int_{\mathbb{R}^{n}}(-f(x)+\lambda u(x)) \phi(x) d x
\end{aligned}
$$

We now deal with the parabolic case and show that $v$ given in (4.5) is the space-distributional solution. We write $v=v_{1}+v_{2}$, where

$$
\begin{aligned}
& v_{1}(t, x)=P_{t} g(x), \\
& v_{2}(t, x)=\int_{0}^{t} P_{t-s} H(s, x) d s, \quad v_{2}(0, \cdot)=0
\end{aligned}
$$

( $v_{1}$ and $v_{2}$ are associated to (4.5) when $H=0$ and $g=0$ respectively). First we deal with $v_{1}$. In [29, Section 4] it is verified that $v_{1}$ is a continuous and bounded function on $[0, \infty) \times \mathbb{R}^{n}$. Furthermore, denoting by $\omega_{g}$ the modulus of continuity of $g$, we have, for any $t \in[0, T], x, y \in \mathbb{R}^{n}$,

$$
\left|P_{t} g(x)-P_{t} g(y)\right| \leq \mathbb{E}\left[\omega_{g}\left(\left|X_{t}^{x}-X_{t}^{y}\right|\right)\right] \leq \omega_{g}\left(|x-y| e^{T L}\right),
$$

where $L=\|A\|_{L}+\|D F\|_{0}$. This shows that $v_{1}(t, \cdot) \in C_{b}\left(\mathbb{R}^{n}\right)$ uniformly in $t \in[0, T]$.

Since (in the classical sense) $\partial_{t}\left(P_{t} f\right)(x)=\mathcal{A}\left(P_{t} f\right)(x)$ for $t>0$ and $x \in \mathbb{R}^{n}$, we see that $t \mapsto \int_{\mathbb{R}^{n}} v_{1}(t, x) \phi(x) d x$ belongs to $C^{1}([0, T])$ and satisfies (4.3) (with $H=0$ ).

Let us deal with $v_{2}$. By the first estimate in (3.9) we deduce, for any $f: \mathbb{R}^{n} \rightarrow \mathbb{R}$ continuous and bounded, and any $h \in\{0, \ldots, k\}$,

$$
\begin{aligned}
\left\|P_{t} f(x+\cdot)\right\|_{C_{b}^{1 /(2 k+1)}\left(E_{h}\left(\mathbb{R}^{n}\right)\right)} & \leq\|f\|_{0}^{1-1 /(2 k+1)}\left\|P_{t} f(x+\cdot)\right\|_{C_{b}^{1}\left(E_{h}\left(\mathbb{R}^{n}\right)\right)}^{1 /(2 k+1)} \\
& \leq C_{1} t^{-1 / 2}\|f\|_{0}
\end{aligned}
$$

for $t \in(0, T]$ and $x \in \mathbb{R}^{n}$, where $C_{1}$ is independent of $t, x$ and $f$. It follows that, for any $x, y \in \mathbb{R}^{n}$ and $t \in[0, T]$,

$$
\begin{aligned}
\left|v_{2}(t, x)-v_{2}(t, y)\right| & \leq \int_{0}^{t} \frac{C_{1}\|H\|_{0}}{(t-s)^{1 / 2}} d s \sum_{h=0}^{k}\left|E_{h}(x-y)\right|^{1 /(2 k+1)} \\
& \leq c^{\prime}\|H\|_{0} \sqrt{T}|x-y|^{1 /(2 k+1)},
\end{aligned}
$$

where $\|H\|_{0}=\sup _{t \in[0, T], x \in \mathbb{R}^{n}}|H(t, x)|$. This shows that $v_{2}(t, \cdot) \in C_{b}\left(\mathbb{R}^{n}\right)$ uniformly in $t$. By this property, in order to verify that $v_{2}$ is continuous 
on $[0, T] \times \mathbb{R}^{n}$, it is enough to check that for any fixed $x \in \mathbb{R}^{n}, v_{2}(\cdot, x)$ is continuous on $[0, T]$. Since the continuity of $v_{2}(\cdot, x)$ at $t=0$ is clear, we consider continuity at a fixed $t \in(0, T]$. We write, for $h$ sufficiently small,

$$
v_{2}(t+h, x)-v_{2}(t, x)=\int_{0}^{T}\left[P_{t+h-s} H(s, x)-P_{t-s} H(s, x)\right] d s
$$

(we have extended $P_{t}$ to negative values, setting $P_{\eta}=0$ for $\eta<0$ ). By the dominated convergence theorem one deduces that $\lim _{h \rightarrow 0} v_{2}(t+h, x)=$ $v_{2}(t, x)$. Thus $v_{2}$ is continuous on $[0, T] \times \mathbb{R}^{n}$ and $v_{2}(0, \cdot)=0$. The boundedness of $v_{2}$ is clear.

It remains to verify that $v_{2}$ satisfies (4.3). To see this, we fix $t \in(0, T]$ and $x \in \mathbb{R}^{n}$, and consider for $h>0$ (see also [28, pp. 58-59])

$$
\frac{v_{2}(t+h, x)-v_{2}(t, x)}{h}=\Gamma_{1}(t, h, x)+\Gamma_{2}(t, h, x),
$$

where

$$
\begin{aligned}
\Gamma_{1}(t, h, x) & =\frac{1}{h} \int_{t}^{t+h} P_{t+h-s} H(s, x) d s, \\
\Gamma_{2}(t, h, x) & =\int_{0}^{t}\left(\frac{P_{t+h-s}-P_{t-s}}{h}\right) H(s, x) d s .
\end{aligned}
$$

We have

$$
\left|\Gamma_{1}(t, h, x)-H(t, x)\right| \leq \int_{0}^{1} \mathbb{E}\left|H\left(t+h-s h, X_{s h}^{x}\right)-H(t, x)\right| d s \rightarrow 0
$$

as $h$ tends to $0^{+}$, by the dominated convergence theorem. It follows that, for any $\phi \in C_{0}^{\infty}\left(\mathbb{R}^{n}\right)$,

$$
\lim _{h \rightarrow 0^{+}} \int_{\mathbb{R}^{n}} \Gamma_{1}(t, h, x) \phi(x) d x=\int_{\mathbb{R}^{n}} H(t, x) \phi(x) d x .
$$

Concerning $\Gamma_{2}$, we first note that, thanks to (4.8), for any $t>s \geq 0$,

$$
\begin{aligned}
\lim _{h \rightarrow 0^{+}} \int_{\mathbb{R}^{n}}\left(\frac{P_{t+h-s} H(s, x)-P_{t-s} H(s, x)}{h}\right) \phi(x) & d x \\
& =\int_{\mathbb{R}^{n}} \mathcal{A}\left[P_{t-s} H(s, \cdot)\right](x) \phi(x) d x .
\end{aligned}
$$

By the Fubini theorem we get 


$$
\begin{aligned}
\lim _{h \rightarrow 0^{+}} \int_{\mathbb{R}^{n}} \Gamma_{2}(t, h, x) \phi(x) d x & =\int_{0}^{t} d s \int_{\mathbb{R}^{n}} P_{t-s} H(s, x) \mathcal{A}^{*} \phi(x) d x \\
& =\int_{\mathbb{R}^{n}} \mathcal{A}^{*} \phi(x) d x \int_{0}^{t} P_{t-s} H(s, x) d s
\end{aligned}
$$

for $t \in(0, T]$. It follows easily that the map $t \mapsto \int_{\mathbb{R}^{n}} v_{2}(t, x) \phi(x) d x$ belongs to $C^{1}([0, T])$ and satisfies $(4.3)$ (with $\left.g=0\right)$ for $t \in[0, T]$.

The next theorems provide elliptic and parabolic Schauder estimates.

THEOREM 4.2. Let $\theta \in(0,1)$ and $\lambda>0$. For any $f \in \mathcal{C}_{d}^{\theta}\left(\mathbb{R}^{n}\right)$ there exists a unique distributional solution to the elliptic equation (4.1). Furthermore, $u \in \mathcal{C}_{d}^{2+\theta}\left(\mathbb{R}^{n}\right)$ and there exists $c=c\left(\lambda, \theta, \nu_{1}, \nu_{2}, A, \tilde{p}, n,\|D F\|_{0},\left\|D^{2} F\right\|_{0}\right.$, $\left.\left\|D^{3} F\right\|_{0}\right)$ such that

$$
\|u\|_{2+\theta, d} \leq c\|f\|_{\theta, d} .
$$

Proof. Uniqueness follows from Theorem 4.1. To prove the assertion we need to investigate the regularity properties of the function $u \in C_{b}\left(\mathbb{R}^{n}\right)$ given in (4.4).

We first prove that $u(z+\cdot) \in C_{b}^{2+\theta}\left(E_{0}\left(\mathbb{R}^{n}\right)\right)$ for any $z \in \mathbb{R}^{n}$, and

$$
\sup _{z \in \mathbb{R}^{n}}\|u(z+\cdot)\|_{C_{b}^{2+\theta}\left(E_{0}\left(\mathbb{R}^{n}\right)\right)} \leq C\|f\|_{\theta, d}
$$

It is clear by the estimates (3.11) that the partial derivatives $D_{i} u$ and $D_{i j}^{2} u$ exist on $\mathbb{R}^{n}$ for any $i, j \in I_{0}=\{1, \ldots, \tilde{p}\}$. Furthermore, $D_{i} u$ and $D_{i j}^{2} u$ are continuous and bounded on $\mathbb{R}^{n}$ and $\left\|D_{i} u\right\|_{0}+\left\|D_{i j}^{2} u\right\|_{0} \leq c\|f\|_{\theta, d}$.

We now prove that $D_{i j}^{2} u \in \mathcal{C}_{d}^{\theta}\left(\mathbb{R}^{n}\right)$ when $i, j \in I_{0}$. This will imply (4.12). To this end, we fix any $v_{h} \in E_{h}\left(\mathbb{R}^{n}\right)$ for $0 \leq h \leq k$ with $\left|v_{h}\right| \leq 1$, and write

$$
\left|D_{i j}^{2} u\left(x+v_{h}\right)-D_{i j}^{2} u(x)\right| \leq u_{1}(x)+u_{2}(x),
$$

where

$$
\begin{aligned}
& u_{1}(x)=\int_{0}^{\left|v_{h}\right|^{2 /(2 h+1)}} e^{-\lambda t}\left|D_{i j}^{2} P_{t} f\left(x+v_{h}\right)-D_{i j}^{2} P_{t} f(x)\right| d t, \\
& u_{2}(x)=\int_{\left|v_{h}\right|^{2 /(2 h+1)}}^{\infty} e^{-\lambda t}\left|D_{i j}^{2} P_{t} f\left(x+v_{h}\right)-D_{i j}^{2} P_{t} f(x)\right| d t,
\end{aligned}
$$

for $x \in \mathbb{R}^{n}$. In order to estimate $u_{1}$ we use $(3.11)(\mathrm{b})$. We find

$$
\sup _{x \in \mathbb{R}^{n}} u_{1}(x) \leq c\|f\|_{\theta, d} \int_{0}^{\left|v_{h}\right|^{2 /(2 h+1)}} t^{\theta / 2-1} d t \leq C\|f\|_{\theta, d}\left|v_{h}\right|^{\theta /(2 h+1)} .
$$


For $u_{2}$ we use estimate (3.11)(c). This gives, for any $x \in \mathbb{R}^{n}$,

$$
\begin{aligned}
\left|D_{i j}^{2} P_{t} f\left(x+v_{h}\right)-D_{i j}^{2} P_{t} f(x)\right| & \leq\left|v_{h}\right| \sup _{r \in I_{h}}\left\|D_{i j r}^{3} P_{t} f\right\|_{0} \\
& \leq c\|f\|_{\theta, d}\left(\frac{1}{t^{(3-\theta) / 2+h}}+1\right)\left|v_{h}\right|
\end{aligned}
$$

for $t>0$. We get

$$
\begin{aligned}
\sup _{x \in \mathbb{R}^{n}} u_{2}(x) & \leq c\|f\|_{\theta, d}\left|v_{h}\right| \int_{\left|v_{h}\right|^{2 /(2 h+1)}}^{\infty} e^{-\lambda t}\left(t^{\theta / 2-3 / 2-h}+1\right) d t \\
& \leq c^{\prime}\left(\left|v_{h}\right| / \lambda+\left|v_{h}\right|^{\theta /(2 h+1)}\right)\|f\|_{\theta, d} \leq C_{1}\|f\|_{\theta, d}\left|v_{h}\right|^{\theta /(2 h+1)} .
\end{aligned}
$$

It follows that $\sup _{x \in \mathbb{R}^{n}}\left|D_{i j}^{2} u\left(x+v_{h}\right)-D_{i j}^{2} u(x)\right| \leq C\|f\|_{\theta, d}\left|v_{h}\right|^{\theta /(2 h+1)}$ and so (4.12) is proved.

We verify that $u(z+\cdot) \in C_{b}^{(2+\theta) /(2 h+1)}\left(E_{h}\left(\mathbb{R}^{n}\right)\right)$ for any $1 \leq h \leq k$, and furthermore

$$
\sup _{z \in \mathbb{R}^{n}}\|u(z+\cdot)\|_{C_{b}^{(2+\theta) /(2 h+1)}\left(E_{h}\left(\mathbb{R}^{n}\right)\right)} \leq C\|f\|_{\theta, d} .
$$

We fix $v_{h} \in E_{h}\left(\mathbb{R}^{n}\right)$ for $1 \leq h \leq k$, with $\left|v_{h}\right| \leq 1$, and compute

$$
\left|u\left(x+v_{h}\right)-u(x)\right| \leq \int_{0}^{\infty} e^{-\lambda t}\left|P_{t} f\left(x+v_{h}\right)-P_{t} f(x)\right| d t=u_{1}(x)+u_{2}(x),
$$

where

$$
\begin{aligned}
& u_{1}(x)=\int_{0}^{\left|v_{h}\right|^{2 /(2 h+1)}} e^{-\lambda t}\left|P_{t} f\left(x+v_{h}\right)-P_{t} f(x)\right| d t, \\
& u_{2}(x)=\int_{\left|v_{h}\right|^{2 /(2 h+1)}}^{\infty} e^{-\lambda t}\left|P_{t} f\left(x+v_{h}\right)-P_{t} f(x)\right| d t,
\end{aligned}
$$

for $x \in \mathbb{R}^{n}$. In order to estimate $u_{1}$ we use $(3.11)(\mathrm{d})$. We find

$$
\sup _{x \in \mathbb{R}^{n}} u_{1}(x) \leq c\|f\|_{\theta, d}\left|v_{h}\right|^{\theta /(2 h+1)} \int_{0}^{\left|v_{h}\right|^{2 /(2 h+1)}} d t \leq C\|f\|_{\theta, d}\left|v_{h}\right|^{(2+\theta) /(2 h+1) .} .
$$

For $u_{2}$ we use $(3.11)($ a) to get (recall that $h \geq 1$ )

$$
\begin{aligned}
\sup _{x \in \mathbb{R}^{n}} u_{2}(x) & \leq c\|f\|_{\theta, d}\left|v_{h}\right| \int_{\left|v_{h}\right|^{2 /(2 h+1)}}^{\infty} e^{-\lambda t}\left(t^{\theta / 2-1 / 2-h}+1\right) d t \\
& \leq C_{1}\|f\|_{\theta, d}\left|v_{h}\right|^{(2+\theta) /(2 h+1)},
\end{aligned}
$$

and (4.14) follows. The proof is complete. 
Theorem 4.3. Let $\theta \in(0,1), T>0, g \in C_{d}^{2+\theta}\left(\mathbb{R}^{n}\right)$ and let $H:[0, T] \times$ $\mathbb{R}^{n} \rightarrow \mathbb{R}$ be a continuous function such that $\sup _{t \in[0, T]}\|H(t, \cdot)\|_{\theta, d}<\infty$. Then the Cauchy problem (4.2) has a unique space-distributional solution $v$ such that $v(t, \cdot) \in C_{d}^{2+\theta}\left(\mathbb{R}^{n}\right)$ for all $t \in[0, T]$. Furthermore, $D_{i} v$ and $D_{i j}^{2} v$ are continuous on $[0, T] \times \mathbb{R}^{n}$ for $i, j \in I_{0}$, and there exists $c=$ $c\left(T, \theta, \nu_{1}, \nu_{2}, A, \tilde{p}, n,\|D F\|_{0},\left\|D^{2} F\right\|_{0}\left\|D^{3} F\right\|_{0}\right)$ such that

$$
\sup _{t \in[0, T]}\|v(t, \cdot)\|_{2+\theta, d} \leq c\left(\|g\|_{2+\theta, d}+\sup _{t \in[0, T]}\|H(t, \cdot)\|_{\theta, d}\right) .
$$

Proof. Uniqueness follows from Theorem 4.1. To prove the result, we need to investigate the space-regularity of the function $v$ given in (4.5); we write $v=v_{1}+v_{2}$ as in (4.9).

For $v_{1}=P_{t} g$ the estimate (3.10)(iv) with $\gamma=2+\theta$ gives immediately (4.16) with $v$ replaced by $v_{1}$ and $H=0$. In order to deal with

$$
v_{2}(t, x)=\int_{0}^{t} \mathbb{E}\left[H\left(s, X_{t-s}^{x}\right)\right] d s=\int_{0}^{t} \mathbb{E}\left[H\left(t-s, X_{s}^{x}\right)\right] d s, \quad t \in[0, T], x \in \mathbb{R}^{n},
$$

we proceed as in the proof of Theorem 4.2. Set

$$
\|H\|_{T, \theta}=\sup _{t \in[0, T]}\|H(t, \cdot)\|_{\theta, d} .
$$

We first prove that $v_{2}(t, z+\cdot) \in C_{b}^{2+\theta}\left(E_{0}\left(\mathbb{R}^{n}\right)\right)$ for $t \in[0, T]$ and $z \in \mathbb{R}^{n}$, and that

$$
\sup _{t \in[0, T], z \in \mathbb{R}^{n}}\left\|v_{2}(t, z+\cdot)\right\|_{C_{b}^{2+\theta}\left(E_{0}\left(\mathbb{R}^{n}\right)\right)} \leq C\|H\|_{T, \theta} .
$$

It is clear from (3.11) that the spatial partial derivatives $D_{i} v_{2}$ and $D_{i j}^{2} v_{2}$ exist on $[0, T] \times \mathbb{R}^{n}$ for any $i, j \in I_{0}$. Furthermore, $D_{i} v_{2}(t, \cdot)$ and $D_{i j}^{2} v_{2}(t, \cdot)$ are continuous and bounded on $\mathbb{R}^{n}$, and $\left\|D_{i} v_{2}(t, \cdot)\right\|_{0}+\left\|D_{i j}^{2} v_{2}(t, \cdot)\right\|_{0} \leq c\|H\|_{T, \theta}$ for any $t \in[0, T]$.

To prove (4.17), we fix $v_{h} \in E_{h}\left(\mathbb{R}^{n}\right)$ for $0 \leq h \leq k$, with $\left|v_{h}\right| \leq 1$, and compute as in (4.13), for any $x \in \mathbb{R}^{n}$,

$$
\begin{aligned}
& \left|D_{i j}^{2} v_{2}\left(t, x+v_{h}\right)-D_{i j}^{2} v_{2}(t, x)\right| \\
& \quad \leq \int_{0}^{t}\left|D_{i j}^{2} P_{s} H\left(t-s, x+v_{h}\right)-D_{i j}^{2} P_{s} H(t-s, x)\right| d s \\
& \quad \leq c\|H\|_{T, \theta} \int_{0}^{t \wedge\left|v_{h}\right|^{2 /(2 h+1)}} s^{\theta / 2-1} d s+c_{1}\|H\|_{T, \theta}\left|v_{h}\right| \int_{t \wedge\left|v_{h}\right|^{2 /(2 h+1)}}^{t} s^{\theta / 2-3 / 2-h} d s \\
& \quad \leq c^{\prime}\|H\|_{T, \theta}\left|v_{h}\right|^{\theta /(2 h+1)},
\end{aligned}
$$


proving (4.17). In order to verify that $v_{2}(t, z+\cdot) \in C_{b}^{(2+\theta) /(2 h+1)}\left(E_{h}\left(\mathbb{R}^{n}\right)\right)$ for any $1 \leq h \leq k$ and $t \in[0, T]$, and

$$
\sup _{z \in \mathbb{R}^{n}, t \in[0, T]}\left\|v_{2}(t, z+\cdot)\right\|_{C_{b}^{(2+\theta) /(2 h+1)}\left(E_{h}\left(\mathbb{R}^{n}\right)\right)} \leq C\|H\|_{T, \theta},
$$

we proceed as in (4.15).

To prove the continuity of $D_{i} v$ and $D_{i j}^{2} v$ on $[0, T] \times \mathbb{R}^{n}$ for $i, j \in I_{0}$, it is enough to show that, for any fixed $x \in \mathbb{R}^{n}, D_{i} v(\cdot, x)$ and $D_{i j}^{2} v(\cdot, x)$ are continuous on $[0, T]$. To see this, we write $x=x_{0}+x_{1}$, where $x_{0}=E_{0} x$ and $x_{1}=x-E_{0} x$, and consider the closed Euclidean ball $K$ in $E_{0}\left(\mathbb{R}^{n}\right)$ centered at $x_{0}$ with radius 1 . We already know that $\left\|v\left(t, x_{1}+\cdot\right)\right\|_{C^{2+\theta}(K)} \leq C_{T}$ for any $t \in[0, T]$. Using the continuity of $v$ on $[0, T] \times \mathbb{R}^{n}$ and a standard compactness argument we obtain the assertion. Note that in particular $\lim _{t \rightarrow 0^{+}} D_{i} v(t, x)=D_{i} g(x)$ and $\lim _{t \rightarrow 0^{+}} D_{i j}^{2} v(t, x)=D_{i j}^{2} g(x)$ for all $x \in \mathbb{R}^{n}$.

5. Schauder estimates with variable $\left(q_{i j}\right)$. Here we study a generalization of the operator $\mathcal{A}$. Namely, we deal with the operator $\tilde{\mathcal{A}}$ in which the diffusion matrix $Q$ depends continuously on $x$, i.e.,

$$
\tilde{\mathcal{A}} u(x)=\frac{1}{2} \operatorname{Tr}\left(Q(x) D^{2} u(x)\right)+\langle A x, D u(x)\rangle+\langle F(x), D u(x)\rangle
$$

for $x \in \mathbb{R}^{n}$. Thanks to the results of Section 4, we will obtain elliptic and parabolic Schauder estimates for the operator $\tilde{\mathcal{A}}$, using a standard approach. This is based on the maximum principle, a priori estimates and the continuity method (cf. [21, Section 6]).

Hypothesis 5.1. (i) There exist $\nu>0$ and an integer $\tilde{p}, 1 \leq \tilde{p} \leq n$, such that the symmetric matrix $Q(x)=\left(q_{i j}(x)\right)_{i, j=1}^{n}$ has the form

$$
Q(x)=\left(\begin{array}{cc}
Q_{0}(x) & 0 \\
0 & 0
\end{array}\right), \quad x \in \mathbb{R}^{n},
$$

where $Q_{0}(x)$ is a uniformly positive definite $\tilde{p} \times \tilde{p}$ matrix such that

$$
\nu \sum_{i=1}^{\tilde{p}} \xi_{i}^{2} \leq \sum_{i, j=1}^{\tilde{p}} q_{i j}(x) \xi_{i} \xi_{j} \leq \frac{1}{\nu} \sum_{i=1}^{\tilde{p}} \xi_{i}^{2}, \quad \xi=\left(\xi_{i}\right) \in \mathbb{R}^{\tilde{p}}, x \in \mathbb{R}^{n} .
$$

(ii) The vector field $F: \mathbb{R}^{n} \rightarrow \mathbb{R}^{n}$ satisfies (ii) and (iii) of Hypothesis 1.1.

(iii) Assumption (iv) of Hypothesis 1.1 holds.

(iv) There exists $\theta \in(0,1)$ such that $q_{i j} \in \mathcal{C}_{d}^{\theta}\left(\mathbb{R}^{n}\right)$ for $i, j \in\{1, \ldots, \tilde{p}\}$, and furthermore, the following limit exists:

$$
\lim _{|x| \rightarrow \infty} Q_{0}(x)=Q_{0}^{\infty} \quad \text { in } L\left(\mathbb{R}^{\tilde{p}}\right) .
$$


Let us comment on these assumptions. Note that, for every $x_{0} \in \mathbb{R}^{n}$, the operator with frozen second order coefficients

$$
\mathcal{A}\left(x_{0}\right)=\frac{1}{2} \operatorname{Tr}\left(Q\left(x_{0}\right) D^{2} \cdot\right)+\langle F(x)+A x, D \cdot\rangle
$$

satisfies Hypothesis 1.1, and therefore the conclusions of Theorems 4.2 and 4.3 hold for $\mathcal{A}\left(x_{0}\right)$. The same happens for the operator $\mathcal{A}^{\infty}$ defined as in (5.5) but with $Q\left(x_{0}\right)$ replaced by $Q^{\infty}\left(Q^{\infty}\right.$ is the $n \times n$ matrix having $Q_{0}^{\infty}$ in the first $\tilde{p} \times \tilde{p}$ block, and zero entries in the other blocks; clearly its coefficients $q_{i j}^{\infty}$ satisfy (5.3)).

To prove the next theorems it is crucial to note that the constants in the elliptic and parabolic Schauder estimates involving $\mathcal{A}\left(x_{0}\right)$ do not depend on $x_{0} \in \mathbb{R}^{n}$.

TheOREM 5.2. Consider the operator $\tilde{\mathcal{A}}$ as in (5.1) under Hypothesis 5.1. Then, for every $\lambda>0$ and $f \in \mathcal{C}_{d}^{\theta}\left(\mathbb{R}^{n}\right)$, the elliptic problem

$$
\lambda u-\tilde{\mathcal{A}} u=f
$$

has a unique solution $u \in \mathcal{C}_{d}^{2+\theta}\left(\mathbb{R}^{n}\right)$ (the first order term $\langle A x, D u(x)\rangle$ in (5.1) is understood in the distributional sense). Furthermore, there exists $c>0$, independent of $f$ and $u$, such that the Schauder estimates (4.11) hold for (5.6).

Proof. We will only sketch the proof, which is not difficult. One needs first a maximum principle for (5.6). We explain how this can be obtained arguing as in the proof of Theorem 4.1. We write $\tilde{\mathcal{A}}=\mathcal{A}_{1}+\mathcal{A}_{2}$, where

$$
\mathcal{A}_{1}=\frac{1}{2} \operatorname{Tr}\left(Q(x) D^{2} \cdot\right) \quad \text { and } \quad \mathcal{A}_{2}=\langle F(x)+A x, D \cdot\rangle .
$$

Take any $u \in \mathcal{C}_{d}^{2+\theta}\left(\mathbb{R}^{n}\right)$ which solves (5.6). Consider a sequence $\left(\rho_{m}\right)$ of mollifiers and set $u_{m}=u * \rho_{m}$; we get, similarly to (4.6),

$$
\begin{aligned}
\tilde{\mathcal{A}} u_{m}(x)= & \int_{\mathbb{R}^{n}} \mathcal{A}_{1} u(x-y) \rho_{m}(y) d y+\int_{\mathbb{R}^{n}} u(y) \mathcal{A}_{2}^{*}\left[\rho_{m}(x-\cdot)\right](y) d y \\
& +R_{m, 1}(x)+R_{m, 2}(x)
\end{aligned}
$$

for $x \in \mathbb{R}^{n}$ and $m \in \mathbb{N}$, where $\mathcal{A}_{2}^{*}$ is the formal adjoint of $\mathcal{A}_{2}$. One finds that $\tilde{\mathcal{A}} u_{m}$ converges in $C_{b}\left(\mathbb{R}^{n}\right)$ to $\tilde{\mathcal{A}} u$ as $m \rightarrow \infty$. By the classical maximum principle (see [3]) we deduce that $\left\|u_{m}\right\|_{0} \leq(1 / \lambda)\left\|\lambda u_{m}-\tilde{\mathcal{A}} u_{m}\right\|_{0}$. Letting $m \rightarrow \infty$, we find $\|u\|_{0} \leq(1 / \lambda)\|\lambda u-\tilde{\mathcal{A}} u\|_{0}$.

A priori estimates for (5.6) can be proved exactly as in the proof of [21, Theorem 8.1]. One assumes that $u \in \mathcal{C}_{d}^{2+\theta}\left(\mathbb{R}^{n}\right)$ is a solution to (5.6) and then by using a localization argument and the maximum principle one finds that there exists $C>0$ (independent of $f$ and $u$ ) such that

$$
\|u\|_{2+\theta, d} \leq C\|f\|_{\theta, d} .
$$


The continuity method allows one to conclude the proof. For any $\epsilon \in[0,1]$ one considers the problem

$$
\lambda u-(1-\epsilon) \mathcal{A}^{\infty} u-\epsilon \tilde{\mathcal{A}} u=f,
$$

where $(1-\epsilon) \mathcal{A}^{\infty} u(x)+\epsilon \tilde{\mathcal{A}} u(x)=\frac{1}{2} \operatorname{Tr}\left(\left[(1-\epsilon) Q^{\infty}+\epsilon Q(x)\right] D^{2} u(x)\right)+\langle F(x)+$ $A x, D u(x)\rangle$. Using the previous a priori estimates, it is straightforward to verify that the set of all $\epsilon$ 's such that (5.8) is uniquely solvable in $\mathcal{C}_{d}^{2+\theta}\left(\mathbb{R}^{n}\right)$ is non-empty, closed and open in $[0,1]$. Taking $\epsilon=1$ in (5.8) one finishes the proof.

In order to state and prove Schauder estimates for the parabolic Cauchy problem involving $\tilde{\mathcal{A}}$, we define the space $\mathcal{C}_{T, d}^{\gamma}, \gamma \in(0,3)$ non-integer. It consists of all continuous functions $v:[0, T] \times \mathbb{R}^{n} \rightarrow \mathbb{R}$ such that $v(t, \cdot) \in$ $\mathcal{C}_{d}^{\gamma}\left(\mathbb{R}^{n}\right)$ for all $t \in[0, T]$, and furthermore $\sup _{t \in[0, T]}\|v(t, \cdot)\|_{\mathcal{C}_{d}^{\gamma}\left(\mathbb{R}^{n}\right)}<\infty$. It is a Banach space endowed with the norm

$$
\|v\|_{\gamma, T, d}=\sup _{t \in[0, T]}\|v(t, \cdot)\|_{\mathcal{C}_{d}^{\gamma}\left(\mathbb{R}^{n}\right)}, \quad v \in \mathcal{C}_{T, d}^{\gamma} .
$$

A function $v \in \mathcal{C}_{T, d}^{2+\theta}, \theta \in(0,1)$, solves the Cauchy problem (4.2) involving $\tilde{\mathcal{A}}$ if $v(0, x)=g(x)$ for all $x \in \mathbb{R}^{n}$, and, for any $\phi \in C_{0}^{\infty}\left(\mathbb{R}^{n}\right)$, the real function $t \mapsto \int_{\mathbb{R}^{n}} v(t, x) \phi(x) d x$ is continuously differentiable on $[0, T]$ and satisfies, for any $t \in[0, T]$ (see (5.7)),

$$
\begin{aligned}
\frac{d}{d t}\left(\int_{\mathbb{R}^{n}} v(t, x) \phi(x) d x\right)= & \int_{\mathbb{R}^{n}} \mathcal{A}_{1} v(t, x) \phi(x) d x+\int_{\mathbb{R}^{n}} v(t, x) \mathcal{A}_{2}^{*} \phi(x) d x \\
& +\int_{\mathbb{R}^{n}} H(t, x) \phi(x) d x .
\end{aligned}
$$

Theorem 5.3. Consider the operator $\tilde{\mathcal{A}}$ in (5.1) under Hypothesis 5.1. Let $T>0, g \in \mathcal{C}_{d}^{2+\theta}\left(\mathbb{R}^{n}\right)$ and $H \in \mathcal{C}_{T, d}^{\theta}$. Then there exists a unique solution $v \in \mathcal{C}_{T, d}^{2+\theta}$ to the Cauchy problem (4.2) involving $\tilde{\mathcal{A}}$. Furthermore, the partial derivatives $D_{i} v$ and $D_{i j}^{2} v$ are continuous on $[0, T] \times \mathbb{R}^{n}$ for $i, j \in I_{0}$, and there exists $c>0$, independent of $g, H$ and $v$, such that

$$
\|v\|_{2+\theta, T, d} \leq c\left(\|g\|_{2+\theta, d}+\|H\|_{\theta, T, d}\right) .
$$

Proof. The proof is similar to the one of Theorem 5.2. Let $v \in \mathcal{C}_{T, d}^{2+\theta}$ be a solution. One first proves the following maximum principle:

$$
\sup _{t \in[0, T], x \in \mathbb{R}^{n}}|v(t, x)| \leq T \sup _{t \in[0, T], x \in \mathbb{R}^{n}}|H(t, x)|+\|g\|_{0},
$$

arguing as in (4.7) (using the fact that $\tilde{\mathcal{A}}=\mathcal{A}_{1}+\mathcal{A}_{2}$ as in the proof of Theorem 5.2).

Concerning the localization procedure which gives the required a priori estimates, we only note that, for any $\eta \in C_{0}^{\infty}\left(\mathbb{R}^{n}\right)$, according to (5.9), the 
function $v \eta$ solves

$$
\left\{\begin{aligned}
\partial_{t}(v \eta)(t, x)= & \tilde{\mathcal{A}}(\eta v)(t, x)-v(t, x) \tilde{\mathcal{A}} \eta(x) \\
& -\langle Q(x) D \eta(x), D v(t, x)\rangle+H(t, x) \eta(x), \quad t \in(0, T], \\
(\eta v)(0, x)= & \eta(x) g(x), \quad x \in \mathbb{R}^{n} .
\end{aligned}\right.
$$

Finally, the continuity method of Theorem 5.2 works also in this case, upon replacing the space $\mathcal{C}_{d}^{2+\theta}\left(\mathbb{R}^{n}\right)$ with $\mathcal{C}_{T, d}^{2+\theta}$, and gives the assertion.

REMARK 5.4. One can weaken the assumption (ii) in Hypothesis 5.1 about $F$ in order to prove elliptic and parabolic Schauder estimates for $\tilde{\mathcal{A}}$. Indeed, we can consider $F: \mathbb{R}^{n} \rightarrow \mathbb{R}^{n}$ such that $F(x)=\left(F_{1}(x), \ldots, F_{\tilde{p}}(x)\right.$, $0, \ldots, 0)$ for $x \in \mathbb{R}^{n}$, and furthermore, there exist $\theta \in(0,1)$ and $M>0$ such that, for any $x, y \in \mathbb{R}^{n}$, if $|y| \leq 1$ then

$$
|F(x)-F(x+y)| \leq M\|y\|^{\theta} .
$$

We briefly explain how to prove elliptic Schauder estimates for $\tilde{\mathcal{A}}$ when $F$ satisfies these assumptions (parabolic estimates are obtained similarly). First we deal with the maximum principle. Let $u \in \mathcal{C}_{d}^{2+\theta}\left(\mathbb{R}^{n}\right)$ be a solution. We consider $u_{m}=u * \rho_{m}$, where $\left(\rho_{m}\right)$ are mollifiers. Under the new assumptions on $F$ one can only show that $\tilde{\mathcal{A}} u_{m}$ converges to $\tilde{\mathcal{A}} u$ uniformly on compact sets of $\mathbb{R}^{n}$ (cf. the proof of Theorem 5.2). This allows us to prove that if $x_{0}$ is a local maximum for $u$ then $\tilde{\mathcal{A}} u\left(x_{0}\right) \leq 0$ (see the proof of [23, Proposition 3.1.10]). Thus the maximum principle holds for $\tilde{\mathcal{A}}$. Then, in order to get Schauder estimates, one writes

$$
\begin{aligned}
\lambda u(x)-\frac{1}{2} \operatorname{Tr}\left(Q(x) D^{2} u(x)\right)-\langle A x & +(F * \rho)(x), D u(x)\rangle \\
& =f+\langle F(x)-(F * \rho)(x), D u(x)\rangle,
\end{aligned}
$$

where $\rho \in C_{0}^{\infty}\left(\mathbb{R}^{n}\right),\|\rho\|_{L^{1}\left(\mathbb{R}^{n}\right)}=1,0 \leq \rho \leq 1$ and $\rho(x)=\rho(-x)$ for $x \in \mathbb{R}^{n}$, and $\rho(x)=0$ if $|x| \geq 1$. Using the formula $D_{i}(F * \rho)(x)=\int_{\mathbb{R}^{n}}(F(x-y)-$ $F(x)) D_{i} \rho(y) d y$ and similar formulae for higher partial derivatives, we see that $F * \rho$ satisfies (iii) of Hypothesis 1.1. Furthermore, by (5.11) one checks that $F-(F * \rho)$ belongs to $\mathcal{C}_{d}^{\theta}\left(\mathbb{R}^{n}\right)$. Finally, easy computations yield Schauder estimates for $\tilde{\mathcal{A}}$.

Acknowledgements. This research was partially supported by the M.I.U.R. research projects Prin 2004 and 2006 "Kolmogorov equations".

\section{References}

[1] S. R. Athreya, R. F. Bass and E. A. Perkins, Hölder norm estimates for elliptic operators on finite and infinite-dimensional spaces, Trans. Amer. Math. Soc. 357 (2005), 5001-5029. 
[2] D. Azé and O. Cärjä, Fast controls and minimum time, Control Cybernet. 29 (2000), 887-894.

[3] M. Bertoldi and L. Lorenzi, Analytical Methods for Markov Semigroups, Chapman \& Hall/CRC, 2007.

[4] J. M. Bismut, Large Deviations and the Malliavin Calculus, Progr. Math. 45, Birkhäuser, 1984.

[5] S. Cerrai, Second Order PDE's in Finite and Infinite Dimensions. A Probabilistic Approach, Lecture Notes in Math. 1762, Springer, 2001.

[6] S. Cerrai and P. Clément, Schauder estimates for a degenerate second order elliptic operator on a cube, J. Differential Equations 242 (2007), 287-321.

[7] G. Da Prato and A. Lunardi, On the Ornstein-Uhlenbeck operator in spaces of continuous functions, J. Funct. Anal. 131 (1995), 94-114.

[8] L. Desvillettes and C. Villani, On the trend to global equilibrium in spatially inhomogeneous entropy-dissipating systems: the linear Fokker-Planck equation, Comm. Pure Appl. Math. 54 (2001), 1-42.

[9] M. Di Francesco and A. Pascucci, On a class of degenerate parabolic equations of Kolmogorov type, Appl. Math. Res. Express 3 (2005), 77-116.

[10] M. Di Francesco and S. Polidoro, Schauder estimates, Harnack inequality and Gaussian lower bound for Kolmogorov type operators in non-divergence form, Adv. Differential Equations 11 (2006), 1261-1320.

[11] F. Flandoli, M. Gubinelli and E. Priola, Well-posedness of the transport equation by stochastic perturbation, preprint, 2008, http://arxiv.org/abs/0809.1310v1.

[12] G. B. Folland, Subelliptic estimates and function spaces on nilpotent Lie groups, Ark. Mat. 13 (1975), 161-207.

[13] M. Freidlin, Some remarks in the Smoluchowski-Kramers approximation, J. Statist. Phys. 117 (2004), 617-634.

[14] M. Fuhrman, Smoothing properties of nonlinear stochastic equations in Hilbert spaces, Nonlinear Differential Equations Appl. 3 (1996), 445-464.

[15] N. V. Krylov, Lectures on Elliptic and Parabolic Equations in Hölder Spaces, Amer. Math. Soc., Providence, 1996.

[16] - Hölder continuity and $L^{p}$ estimates for elliptic equations under general Hörmander's condition, Topol. Methods Nonlinear Anal. 9 (1997), 249-258.

[17] S. Kusuoka and D. W. Stroock, Application of the Malliavin calculus, III, J. Fac. Sci. Univ. Tokyo IA Math. 34 (1987), 391-442.

[18] E. Lanconelli and S. Polidoro, On a class of hypoelliptic evolution operators, Rend. Sem. Mat. Univ. Politec. Torino 52 (1994), 26-63.

[19] L. Lorenzi, Estimates of the derivatives for a class of parabolic degenerate operators with unbounded coefficients in $\mathbb{R}^{N}$, Ann. Scuola Norm. Sup. Pisa Cl. Sci. (5) 4 (2005), 255-293.

[20] - Schauder estimates for degenerate elliptic and parabolic problems with unbounded coefficients in $\mathbb{R}^{N}$, Differential Integral Equations 18 (2005), 531-566.

[21] A. Lunardi, Schauder estimates for a class of degenerate elliptic and parabolic operators with unbounded coefficients, Ann. Scuola Norm. Sup. Pisa 24 (1997), 133-164.

[22] —, An interpolation method to characterize domains of generators of semigroups, Semigroup Forum 53 (1996), 321-329.

[23] —, Analytic Semigroups and Optimal Regularity in Parabolic Problems, Birkhäuser, 1995.

[24] N. Ikeda and S. Watanabe, Stochastic Differential Equations and Diffusion Processes, North-Holland and Kodansha, 1981. 
[25] M. Manfredini, The Dirichlet problem for a class of ultraparabolic equations, Adv. Differential Equations 2 (1997), 831-866.

[26] A. Nagel, E. M. Stein and S. Wainger, Balls and metrics defined by vector fields. I. Basic properties, Acta Math. 155 (1985), 103-147.

[27] A. Pascucci, Hölder regularity for a Kolmogorov equation, Trans. Amer. Math. Soc. 355 (2003), 901-924.

[28] E. Priola, The Cauchy problem for a class of Markov-type semigroups, Comm. Appl. Anal. 5 (2001), 49-75.

[29] — Formulae for the derivatives of degenerate diffusion semigroups, J. Evol. Equations 6 (2006), 577-600.

[30] L. P. Rothschild and E. M. Stein, Hypoelliptic differential operators and nilpotent groups, Acta Math. 137 (1976), 247-320.

[31] N. Saintier, Schauder estimates for degenerate elliptic and parabolic equations in $\mathbb{R}^{n}$ with Lipschitz drift, Differential Integral Equations 20 (2007), 397-428.

[32] T. Seidman, How violent are fast controls?, Control Signals Systems 1 (1988), 89-95.

[33] E. Stein, Harmonic Analysis: Real-Variable Methods, Orthogonality, and Oscillatory Integrals, Princeton Math. Ser. 43, Princeton Univ. Press, 1993.

[34] H. Triebel, Interpolation Theory, Function Spaces, Differential Operators, NorthHolland, Amsterdam, 1978.

[35] J. Zabczyk, Mathematical Control Theory: An Introduction, Birkhäuser, 1992.

Dipartimento di Matematica

Università di Torino

via Carlo Alberto 10

10123 Torino, Italy

E-mail: enrico.priola@unito.it

Received August 14, 2008

Revised version February 20, 2009 